\title{
Evolución de la humedad en presas de relaves mineros ubicadas en climas áridos. Caso de estudio: mina Castaño Viejo, San Juan, República Argentina
}

\author{
Lucas Garino Libardi y Luciano Oldecop \\ Instituto de Investigaciones Antisísmicas, Facultad de Ingeniería, Universidad Nacional de San Juan, Av. Libertador \\ General San Martin Oeste 1290, San Juan, Argentina. \\ Igarino@unsj.edu.ar; oldecop@unsj.edu.ar
}

\begin{abstract}
RESUMEN
Este trabajo presenta los resultados obtenidos en campañas de exploración geotécnica realizados en presas de relaves mineros resultantes del proceso de extracción de plomo, zinc y cobre. Las presas estudiadas se encuentran abandonadas hace más de medio siglo y se ubican en la cordillera de los Andes, San Juan, Argentina. Esta zona, de clima árido, se caracteriza por tener un régimen hídrico deficitario. La observación de grados de saturación sorprendentemente elevados en el interior de los relaves luego de 50 años de libre interacción con la atmósfera, condujo a la realización de un experimento de campo de larga duración (ensayo de parcela) con el objeto de investigar el inusual comportamiento. Sobre la base de los resultados del ensayo se concluye que la estratigrafía tiene una influencia preponderante en la evolución a largo plazo de la cantidad de agua almacenada. Capas arcillosas y limosas presentan grados de saturación elevados por efecto de barrera capilar, mientras que las capas intercaladas de arena se secan llegando a una condición saturación residual. Esta condición provoca que el interior de los relaves quede hidrológicamente aislado de los fenómenos atmosféricos en superficie. Se observó, además, que la profundidad de la capa activa es de 10 a $35 \mathrm{~cm}$ para la evaporación y de $35 \mathrm{a} 75 \mathrm{~cm}$ para la infiltración. Tal asimetría determina un flujo neto de ingreso de agua en el sistema, lo cual es consistente con la generación y conservación de un elevado grado de saturación en el interior de los relaves.
\end{abstract}

Palabras clave: evolución humedad, estratificación, minería, presas de relaves, zona de saturación.

\section{Evolution of moisture in tailings dams located in arid climates. Case study: Castaño Viejo mine, San Juan, Argentina}

\begin{abstract}
This paper presents the results obtained in geotechnical exploration campaigns carried out in mining tailings dams resulting from the extraction process of lead, zinc and copper. The studied dams have been abandoned for more than half a century and are located in the Andes Mountains, San Juan, Argentina, an area characterized by water deficit regimens. The observation of degrees of saturation, which is surprisingly high in the internal zones of the tailings dams, led to a long-term field experiment (plot test) to investigate the observed behaviour. Based on the results of the plots test, we have concluded that stratigraphy controls the evolution of the water stored with the tailings. Clay and silty layers develop high degrees of saturation due to the capillary barrier effect, whilst the interlayers of sand dry up to a residual saturation condition. This situation inhibits the capillary rise and the diffusion of vapour, thus isolating the interior of the tailings from the atmosphere. It was observed that the depth of the active layer is 10 to $35 \mathrm{~cm}$ for evaporation and 35 to $75 \mathrm{~cm}$ for infiltration in the plot experiment. Such asymmetry determines a net flow of water into the system, which is consistent with the generation and conservation of a high degree of saturation within the tailings.
\end{abstract}

Keywords: mining; moisture evolution; saturation zone; stratification; tailings dams. 


\section{ABRIDGED ENGLISH VERSION}

\section{Introduction and methods}

Research on causes of worldwide tailings dam failures concludes that the hydraulic conditions of the contained tailings play a fundamental role in the development of the failure, as well as in the magnitude of the consequences.

In Argentina, the construction and operation of large tailings dams is scarce and relatively recent. In addition, local experience in mine closure is very incipient. Recently there was the first closure of a mine that had been active since 1997 (Bajo de la Lumbrera mine- Catamarca).

In this context, an initiative was taken to study the long-term hydro-mechanical behaviour of small tailings dams that were abandoned about 50 years ago and which since then have been interacting liberally with the atmosphere (Fig. 1).

Trial pits were performed on the CV1 and CV2 tailings dams of the Castaño Viejo mine (Figs. 2 and 3). Significant stratification was observed. The millimetric layers formed by sand particles are covered by other layers where the particles are of silt size (Fig. 4). The surface layer formed by silts with sand intercalations is extremely hard. This layer has patterns of cracks and fissures resulting from shrinkage during drying processes (Fig. 5).

In both dams, the tailings found present very similar particle size curves (Fig. 8).

The analysis of the samples recovered from manual boreholes allowed us to establish simplified stratigraphic profiles and profiles of variation gravimetric moisture with depth (Figs. 6 and 7).

We observed that except for a surface layer of about $1.0 \mathrm{~m}$ deep, the degrees of saturation are high, a situation that is not easy to explain if we take into account that the hydric deficit is very important (potential annual evaporation / annual precipitation $>10$ ).

In the CV1 tailings dam, a long-term field experiment (528 days) was carried out to study the role of stratification in the evolution of the water contained in the unsaturated zone (Fig. 9). In the volume of the isolated tailings, agronomic sensors were installed to measure the variables of interest: volumetric water content, matrix suction and temperature (Fig. 10 and Table 1).

The field experiment was divided into two stages. Phase " $A$ " covers the first 72 hours of testing, in which twelve controlled irrigations were carried out on the surface of the tailings.

Once Phase $A$ was concluded, the surface of the tailings was allowed to interact liberally with the atmosphere for more than a year and a half, Phase " $B$." In addition, a weather station was installed in the proximity of the CV1 tailings dam, where ambient temperature, relative humidity and rainfall were recorded during the test period.

\section{Results and discussion}

The irrigations carried out in Phase $A$ and the summer rains in Phase $B$ of the experiment, constituted water contributions of similar magnitude, although with very different intensities. Graphs show the variation of gravimetric moisture in the tailings for both phases (Fig. 11 to Fig. 13).

All the irrigation and rain events produce a reaction in the surface sensors (S1 and S6), so it is evident that the first $10 \mathrm{~cm}$ of tailings are strongly influenced by atmospheric agents, even their hard consistency and low permeability. The pattern of small cracks and fissures caused by shrinkage may have a decisive influence on the infiltration process.

The response of the rest of the instruments during infiltration processes is very complex. The sensors do not react in an order consistent with a wet front advancing downwards. On the contrary, what is observed is that the order of response has a direct relationship with the type of material where the sensors are installed. For example, during Phase A, sensors 51 (7 $\mathrm{cm}), S 6(9 \mathrm{~cm})$ and $S 8(75 \mathrm{~cm})$, installed in layers of fine particles (silt and clayey silt) react two days earlier than the rest of the sensors $S 2(15 \mathrm{~cm}), S 7(21 \mathrm{~cm})$ and S3 $(35 \mathrm{~cm})$, installed in layers where the particles have larger sizes (fine sand). The fingering phenomenon may explain why the deepest sensor, S8, reacts much earlier than all sensors installed above, but in sand (Figs. 14 and 15).

Surface sensor logs show large daily and seasonal temperature variations (Fig.16), with maximum temperatures in the order of $40^{\circ} \mathrm{C}$ and minimum temperatures below zero to $35 \mathrm{~cm}$ depth. The amplitude of the recorded temperatures decreases with depth. For the deepest sensor (S8 located at $75 \mathrm{~cm}$ ) the maximum temperatures recorded are $25^{\circ} \mathrm{C}$ whilst the minimum is around $10^{\circ} \mathrm{C}$.

During the periods of the experiment without irrigation or rainfall, a gradual decrease in humidity sensor readings was observed. The superficial sensors (S1 and S6) have the typical behaviour of an evaporation process. The intermediate and deep sensors kept fluctuating slightly at around $6 \%$ moisture for about 2 years.

Intermediate sensors do not seem likely to be affected by evaporation, at least permanently; rather, their variations seem to respond to a redistribution of moisture or percolation to the deeper layers.

In any case, it is obvious that the sensors below the surface layer have a drastically lower influence from atmospheric agents.

In principle, it is difficult to think that in an arid environment where the water deficit is very significant (potential annual evaporation / annual precipitation ratio $>10)$ the atmosphere cannot extract moisture from layers located at only $35 \mathrm{~cm}$ depth. However, 
moisture records do not seem to indicate that the layers at that or greater depths are affected by evaporation, at least permanently, and rather it seems that variations in humidity respond to a redistribution of water or percolation to deeper layers. Suction records (Fig. 17) seem to confirm this hypothesis. The S10 sensor $(35 \mathrm{~cm})$ always registers a greater suction than the S4 sensor $(15 \mathrm{~cm})$, making capillary ascent impossible.

The mechanism that could explain this behaviour is also the capillary barrier (Fig. 18). The drying of the sand layers determines that its unsaturated permeability decreases drastically, even losing continuity of the liquid phase, thus becoming barriers for capillary ascent.

The lower layers are isolated from the atmosphere by multiple layers of sand in which the only possible transport is by molecular vapour diffusion, an extremely slow mechanism. If in addition, the higher layers of silt have high degrees of saturation, these constitute a barrier for the flow of gases and the vapour diffusion, which is why the evaporation would be totally impeded.

On the other hand, the experiment suggests that evaporative drying effects do not extend more than 10 to $15 \mathrm{~cm}$ deep. Only when the amount of stored water is high (after rain for example) can the effects of evaporative drying be temporarily extended to a depth greater than $35 \mathrm{~cm}$, but less than $75 \mathrm{~cm}$. Intense oxidation of some thin layers is observed only up to $50 \mathrm{~cm}$ deep, which is an independent indicator of the depth of the "active layer."

\section{Conclusions}

The plot test does not have a simple interpretation due to the complexity originated in the stratification of the tailings. From analysing the registers of the sensors it can be established that the sector of the tailings dam where the processes linked to the transfer of water with the atmosphere take place has a thickness somewhat greater than $35 \mathrm{~cm}$ and less than $75 \mathrm{~cm}$. It is astonishing that the thickness of the "active layer" is so extraordinarily small.

The field experiment shows evidence of heterogeneous flow (fingering) during infiltration processes related to irrigation events. The presence of thin layers of sand alternating with layers of silt forms a barrier system that is extraordinarily effective in preventing water evaporation, both by capillary ascent and by vapour diffusion.

The mechanisms that influence the movement of water in the unsaturated zone of the CV1 tailings dam, especially evaporation, are strongly controlled by the marked stratification, even when the atmospheric conditions generate extremely efficient drying processes.

An asymmetric behaviour is inferred in the interaction of the tailings dam with the atmosphere, since considering all the described mechanisms, the infiltration would reach greater depths than the effects of evaporation.

In the long term, there would be a net entry of water to the tailings dam, which reaching the deep layers could no longer be extracted by the atmosphere.

This asymmetric phenomenon would produce a "greenhouse effect" on the water that enters and leaves the tailings dam. This is consistent with the high degrees of saturation found in the tailings dams studied, even though more than 50 years have passed since they were abandoned.

The discharge of tailings with high water content (sludge) generates open, sparsely dense structures with extremely low penetration resistances (Garino et al., 2017; (GME, 2004).

Given the high degree of saturation inside the tailings, liquefaction can be expected if dynamic actions due to earthquakes of moderate intensity act on these structures.

Therefore, if long-term stability analysis is to be performed, it is appropriate to consider tailings with low resistance and in a condition close to saturation, even when environmental conditions are conducive to evaporation.

If these structures are located in areas with seismic activity, it would be convenient to consider post liquefaction or residual strength parameters in the tailings in order to verify their stability.

\section{Introducción}

Hasta mediados del siglo pasado, la mayor parte de la minería metalífera consistía en pequeñas explotaciones subterráneas de vetas de alta ley y por lo tanto con una generación de residuos moderada. Debido, entre otras cosas, a la creciente demanda de materia prima, a los avances realizados por los fabricantes de máquinas y equipos, y a los desarrollos de nuevas tecnologías de extracción, se ha transformado en rentable la explotación de grandes yacimientos minerales con leyes muy reducidas. Es así que las explotaciones a cielo abierto actualmente generan grandes cantidades de residuos.

De los diversos residuos generados por la actividad minera, los lodos, comúnmente denominados: relaves, colas o tailings, son los más abundantes. Los relaves están constituidos por roca finamente molida, agua y aditivos químicos propios del proceso de extracción. La forma más habitual de disposición de los relaves es el bombeo y vertido por tuberías dentro de un reservorio, lo que en ingeniería civil se conoce como relleno hidráulico. Los relaves vertidos de esta manera requieren de estructuras para su contención, es decir de una presa.

Una presa de relaves (PR) es una estructura formada con los propios relaves tratados de manera conveniente para otorgarles la resistencia mecánica necesaria o con otros materiales de préstamo. Su objetivo es 
el almacenamiento del residuo, por tiempo indefinido, de forma segura y minimizando el impacto sobre el medio ambiente.

La falla de una PR tiene, en muchos casos, consecuencias catastróficas para el medio ambiente, para los trabajadores de la mina y para poblaciones vecinas (Chandler and Tosatti, 1995; Harder and Stewart, 1996; Bligth, 1997; Fourie et al., 2001; Alonso and Gens, 2006; Oldecop y Rodríguez, 2006; Bray and Frost, 2010; IEEIRP, 2015; Morgenstern et al., 2016; Roche et al., 2017). Por otra parte, un abandono en condiciones inadecuadas, puede provocar daños irreversibles a los recursos hídricos, al suelo, la vegetación y fauna del lugar de emplazamiento (García, 2004).

Los casos de rotura de PR recopilados en el Boletín 121 (ICOLD, 2001) y otros trabajos posteriores (Davies, 2002; Bligth and Fourie, 2005), indican que el manejo del agua en estos depósitos es un factor fundamental para su seguridad. Casos históricos de fallas causadas por muy diversos factores y mecanismos (deslizamientos, terremotos, sobrepaso, tubificación y falla de estructuras auxiliares), muestran que, casi siempre, las condiciones de operación hidráulica juegan un papel fundamental en el desarrollo de la falla, así como en la magnitud de sus consecuencias (Oldecop et al., 2008).

Una investigación sobre la base de datos de $147 \mathrm{ca}$ sos de falla en todo el mundo muestra que el $39 \%$ de las roturas de PR se debe a factores combinados entre lluvias intensas, sobrepaso, erosión de taludes y otros factores como una inadecuada gestión y ubicación de la laguna de decantación (Rico et al., 2008).

Análisis recientes de las fallas ocurridas en PR indican que, aunque el número total de fallas ha disminuido, el número de fallas graves ha aumentado en las últimas tres décadas y en la mayoría de los casos las inestabilidades están asociadas a cambios bruscos en las condiciones hidráulicas de los relaves (Bowker and Chambers, 2015, Roche et al., 2017).

El agua almacenada en la zona no saturada de una $P R$, puede también jugar un papel decisivo en la estabilidad del depósito ante aportes extraordinarios de agua provenientes de lluvias, deshielos o crecientes (Zandarín et al., 2009).

En Argentina la experiencia en la construcción y operación de grandes PR es escasa y relativamente reciente: Minera Aguilar (Ag, $\mathrm{Pb}$ y $\mathrm{Zn}$ ), Pirquitas ( $\mathrm{Ag}$ y $\mathrm{Zn}$ ), Cerro Vanguardia, Cerro Negro, Manantial Espejo y Don Nicolás ( $\mathrm{Au}$ y $\mathrm{Ag}$ ), todos ellos en operación.

El precio internacional de algunos metales, como el oro, la plata y el cobre, han justificado planes de prospección y el desarrollo de nuevos proyectos mineros. En la provincia de San Juan se destacan los proyectos: Pascua-Lama, Constelación, Del Carmen, El Pachón, Altares, Los Azules. La mayoría de estos proyectos prevé disponer los relaves en PR con la técnica de relleno hidráulico.

Por otra parte, la experiencia local sobre el cierre de minas es muy incipiente. Recientemente se produjo el primer cierre de una mina que se encontraba activa desde 1997 (Bajo de la Lumbrera - Catamarca).

El objetivo final de la investigación es poder establecer condiciones reales para los relaves, que permitan estimar el comportamiento estructural de PR durante su operación como así también en la etapa de cierre y abandono.

Para poder establecer tales condiciones resulta indispensable conocer la evolución de la humedad en los relaves a corto, mediano y largo plazo. Dicha predicción es difícil de hacer, pues depende de un número importante de factores y fenómenos complejos comprendidos en la interacción de los relaves con su entorno (atmósfera y fundación), involucrando fenómenos tales como: infiltración, evaporación, ascenso capilar, transporte y precipitación de sales, entre otros.

En este contexto resulta interesante aprovechar la existencia de pequeñas $\mathrm{PR}$ abandonadas hace alrededor de 50 años, para ser utilizadas como casos de estudio y testigo del comportamiento hidromecánico a largo plazo, en general, y en particular para analizar la evolución de la humedad de los relaves y los factores que la afectan.

\section{Caso de estudio}

La mina "Castaño Viejo" distante unos $200 \mathrm{~km}$ de la ciudad de San Juan, Argentina, está ubicada en la Cordillera Frontal y próxima a la margen derecha del Río Castaño, afluente del Río San Juan. Esta área fue explotada durante el período 1956-1964, y durante estos años se obtuvieron un total de $46.640 \mathrm{t}$ de concentrados con $73-78 \%$ de $\mathrm{Pb}, 75.588 \mathrm{t}$ con $50-60 \%$ de $\mathrm{Zn}$ y 8.000 t con $14-21 \%$ de Cu (Gramage, 1983).

El tratamiento mineralúrgico empleado fue de trituración, molienda y posterior flotación diferencial. Los relaves compuestos por un 60 a $70 \%$ de agua en peso (Ramírez et al., 2002) y partículas de granulometría fina, fueron trasportados por canales y cañerías por gravedad hasta distintos sitios de almacenamiento. Estos depósitos, construidos por el método de relleno hidráulico y recrecimiento hacia aguas arriba, se encuentran distribuidos en tres zonas, entre los 2340 y 1935 m.s.n.m.

Los estudios geotécnicos presentados en este trabajo se concentran principalmente en una PR ubicada a $5 \mathrm{~km}$ aguas abajo de la planta de tratamiento, denominada CV1. Se muestran también algunos resultados de ensayos realizados en una de las PR del sector central, denominada CV2 (Fig. 1).

La PR CV2 integra una serie de 22 pequeños depósitos que se disponen sobre la margen izquierda de un curso de estiaje temporal. Estas PR presentan un desarrollo longitudinal aproximadamente de $1 \mathrm{~km}$ y alturas que van de los $7 \mathrm{~m}$ a $15 \mathrm{~m}$.

Debido al estado de abandono, las estructuras de contención presentan un deterioro importante generado por la erosión asociada a escorrentías tempora- 


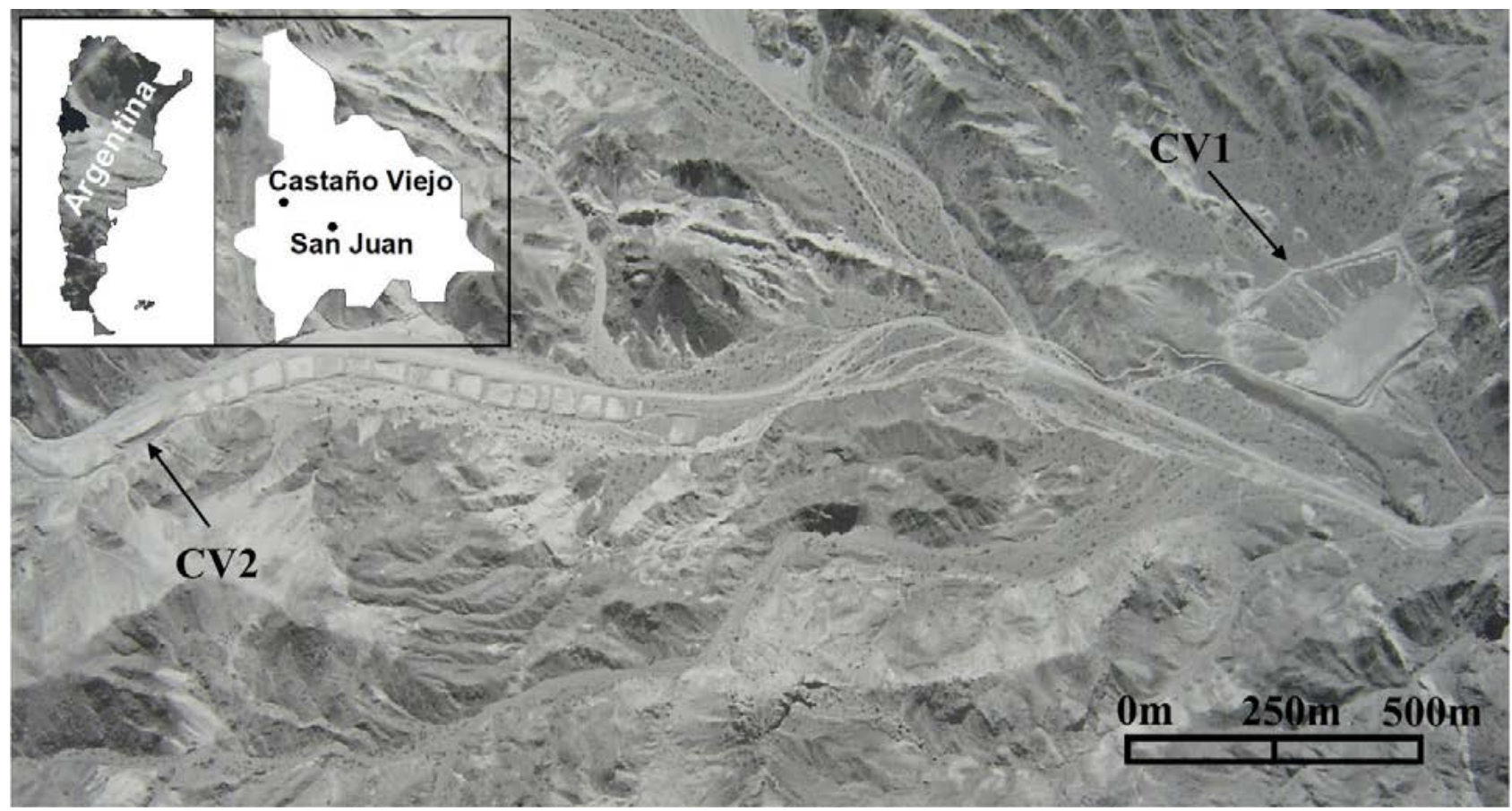

Figura 1. Fotografía aérea de las balsas de lodos estudiadas, CV1 (derecha) y CV2 (izquierda) y. CEFOCCA-UNSJ, año 1960.

Figure 1. Aerial photograph of the distribution of tailings dams studied in the central sector (left) and eastern sector (right). CEFOCCAUNSJ, 1960.

les (Fig. 2).

La PR CV1 es la de mayor tamaño (Fig. 3). Ocupa una superficie de $56.160 \mathrm{~m}^{2}$, sus dimensiones mayores aproximadamente son de $216 \mathrm{~m} \times 345 \mathrm{~m}$, y el coronamiento del cierre presenta un desarrollo de 405 $\mathrm{m}$ con $15 \mathrm{~m}$ de altura máxima.

En todo el perímetro de la PR CV1 hay vestigios de las canaletas utilizadas para la conducción y vertido de los relaves. El método de relleno hidráulico, gene- ra estructuras estratigráficas complejas. Al ser vertidos los relaves desde un punto del perímetro del reservorio, generan una estructura que se asemeja a un abanico aluvial. La granulometría del material depositado disminuye su tamaño desde el perímetro hacia el centro del depósito. En la zona perimetral o zona de vertido, se deposita la fracción de tamaño arenas medias a finas (sedimentación fluvial). A una cierta distancia del perímetro hay una zona de transición,

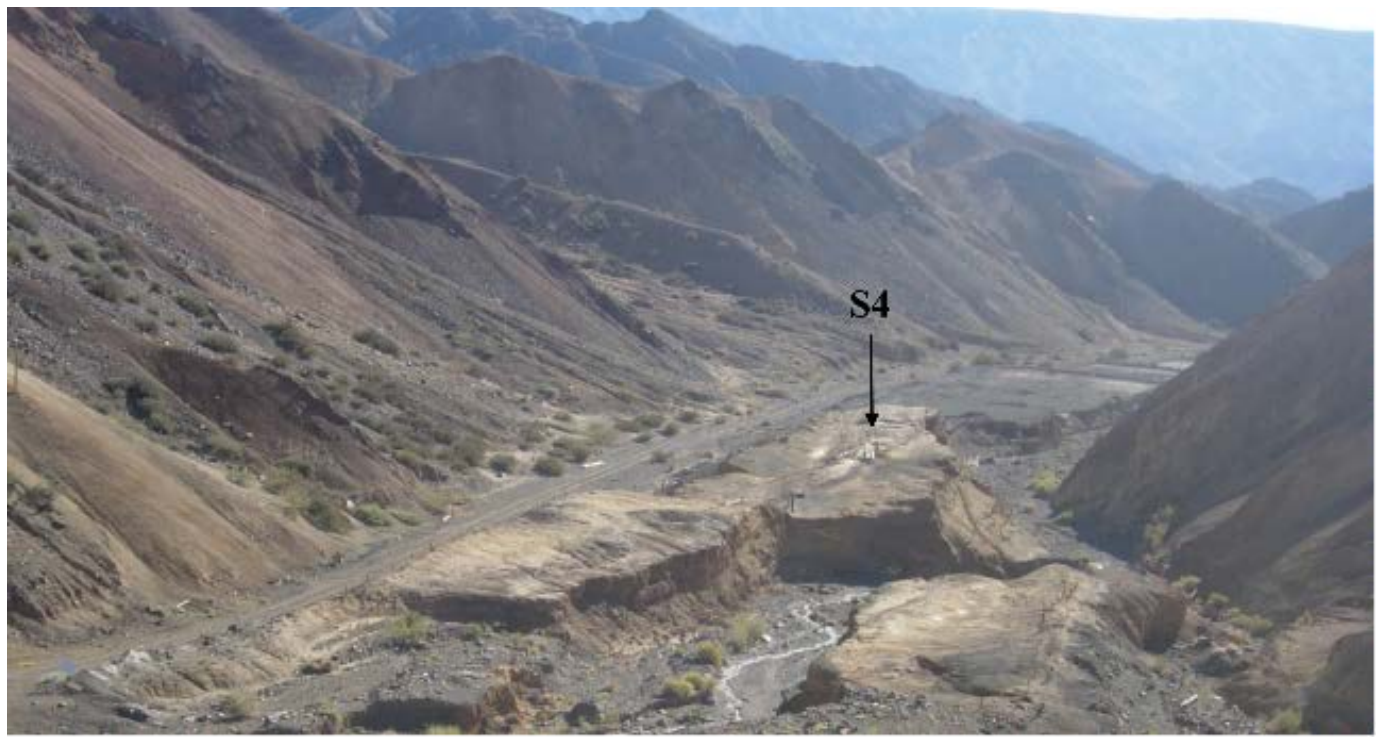

Figura 2. PR CV2, vista hacia el este. S4: sondeo. Marzo de 2009.

Figure 2. Tailings dam CV2, view to the east. S4: exploration borehole. March 2009. 

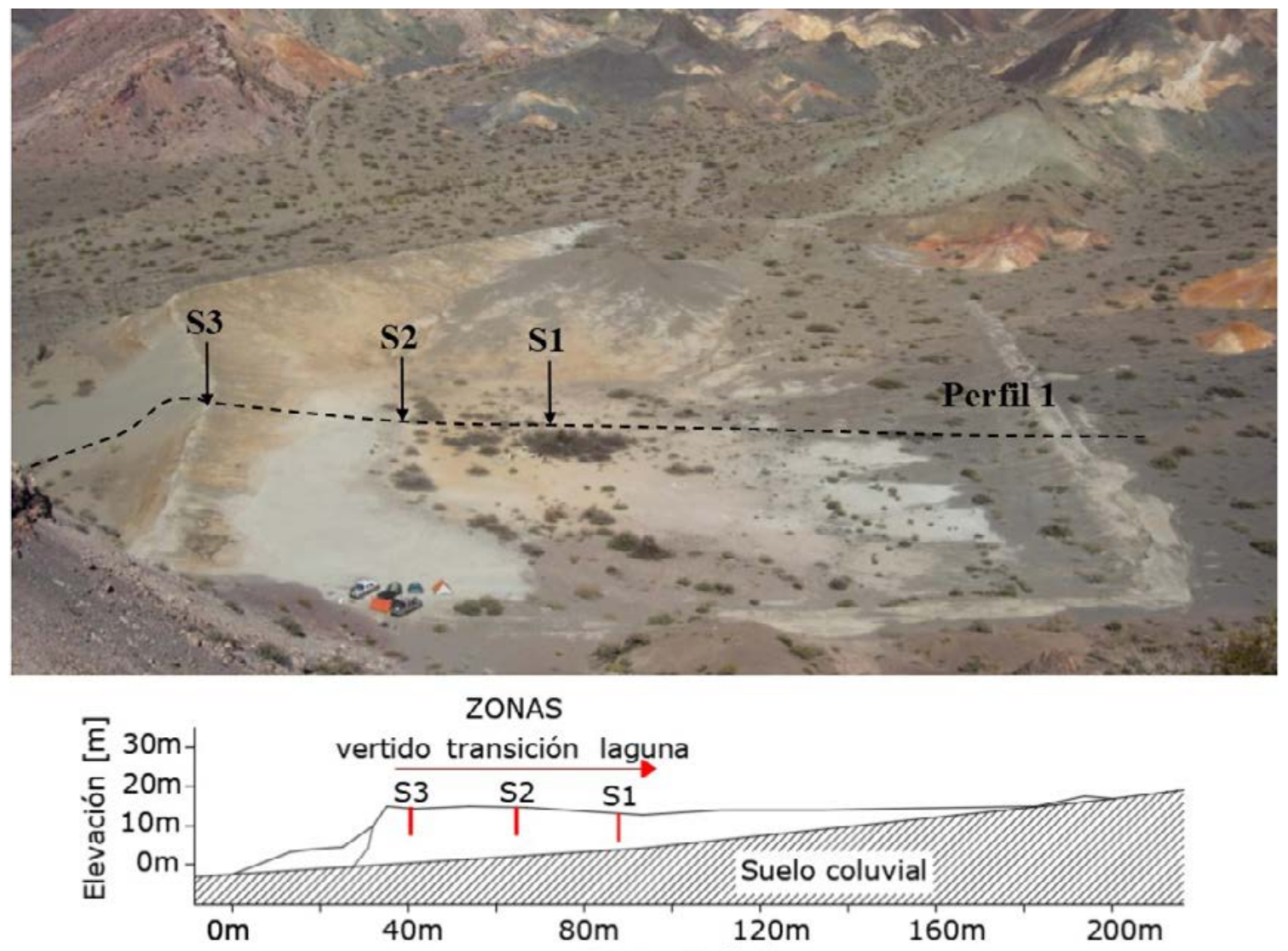

Distancia $[\mathrm{m}]$

Figura 3. PR CV1, vista hacia el oeste (arriba) y perfil topográfico 1 (abajo). S1, S2, S3: ubicación de sondeos. Octubre de 2009.

Figure 3. Tailings dam CV1, view to the west. S1, S2, S3: exploration boreholes. October 2009.

con partículas de tamaño intermedio, arenas finas y limos. En el centro del depósito se acumula el líquido y la fracción más fina, limos y arcillas, formando la laguna de decantación (sedimentación lacustre). Esta clasificación espacial de tamaños de partícula influye en la permeabilidad de los relaves. En el caso de estudio, los coeficientes de permeabilidad varían en un orden de magnitud entre la zona perimetral y el centro del depósito (Garino et al, 2017). Para lograr un crecimiento uniforme en altura de la presa, el punto de vertido se desplaza periódicamente a lo largo del perímetro. Esto produce la superposición de los abanicos y de capas de relaves de distinta granulometría, dando lugar a perfiles estratificados. En las calicatas realizadas en ambas PR de Castaño Viejo, se observaron capas alternadas de arena, limo y arcilla. En algunos niveles, estos estratos tienen espesores notablemente pequeños, algunos de tan solo un par de milímetros (Fig. 4).

La capa superficial de las PR estudiadas $(5$ a $10 \mathrm{~cm}$ de profundidad), donde predominan los estratos limosos con intercalaciones de pequeños estratos de

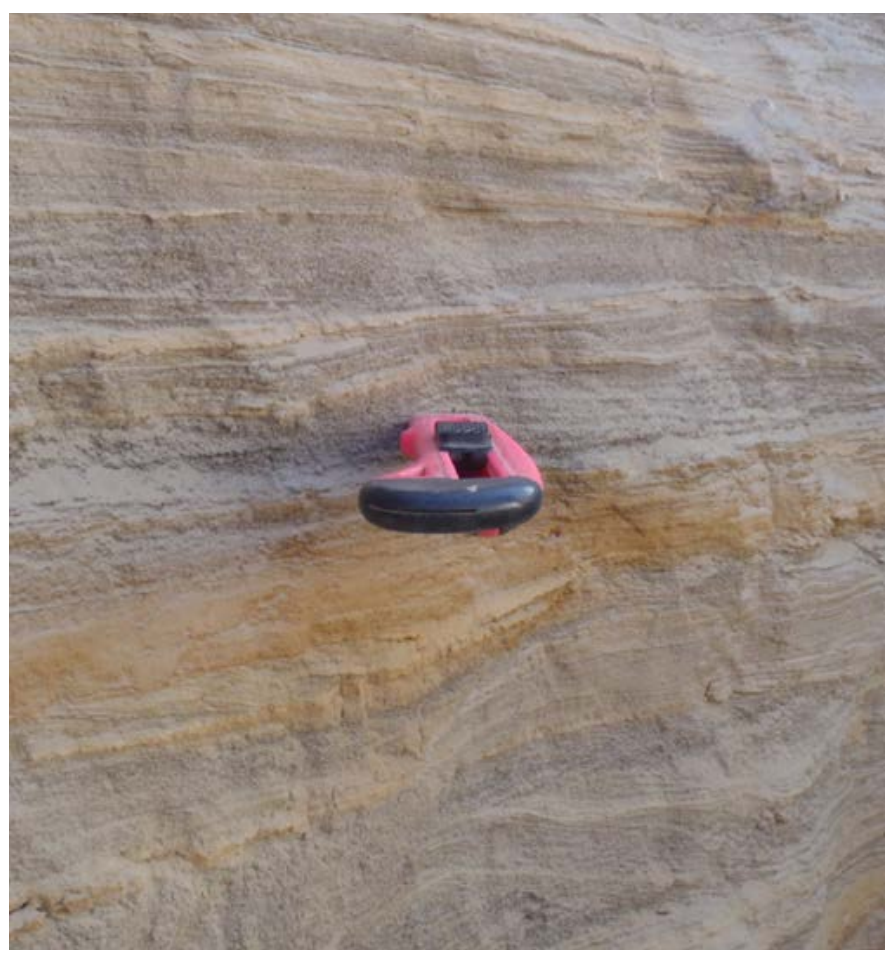

Figura 4. Calicata realizada en la CV1. Alternancia de finos estratos de limo y arena fina. Octubre de 2012.

Figure 4. Trial pit in CV1 tailings dam. Sequence of thin layers of silt and fine sand. October 2012. 

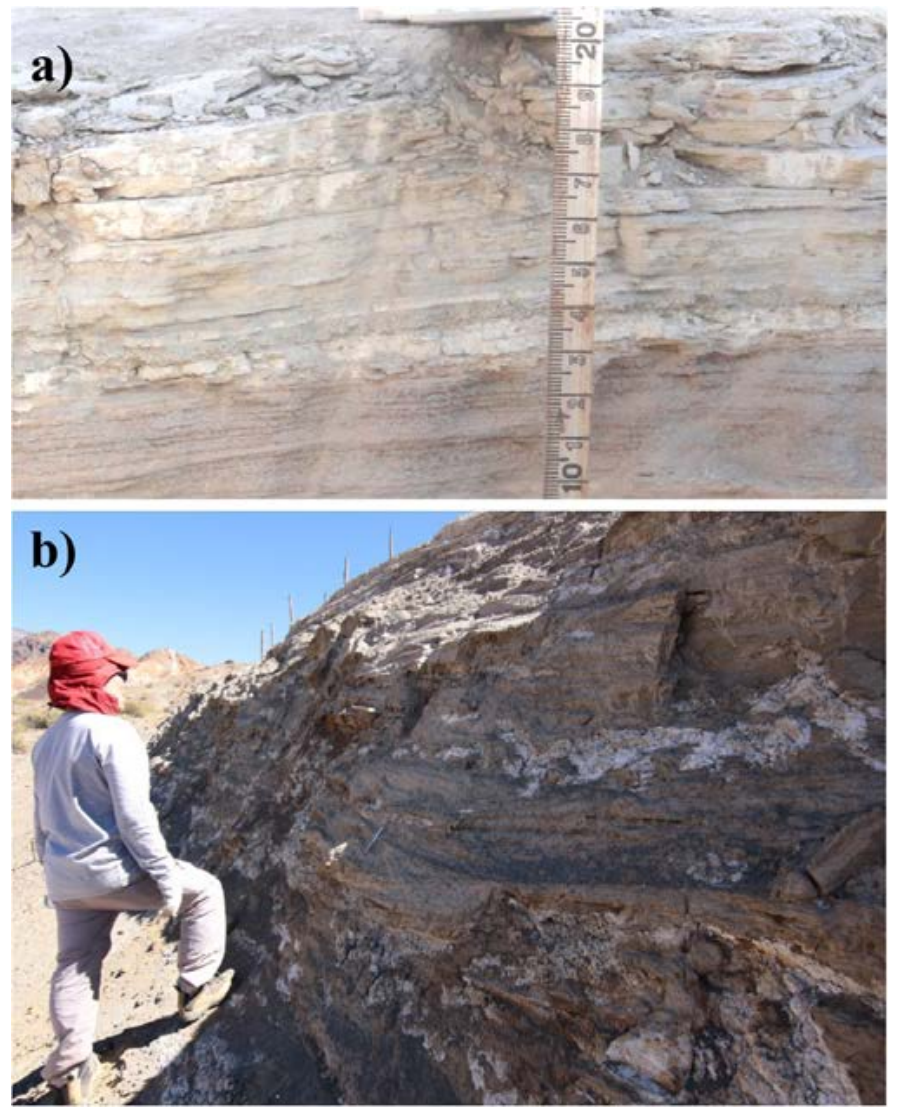

Figura 5. a) Estratos superficiales duros y agrietados. b) Eflorescencias salinas en el talud frontal de la CV1. Marzo de 2018. Figure 5. a) Hard and cracked superficial layers. b) Saline efflorescence in the frontal slope of the CV1 tailings dam. March 2018. arena, presenta alteraciones físico-químicas producto del contacto con los agentes atmosféricos. Resulta una capa extremadamente dura con valores de permeabilidad cercanos a una arcilla $(9,75 E-07 \mathrm{~m} / \mathrm{s})$. Se observan también grietas o fisuras producto de la retracción durante el secado. Estas grietas tienen un desarrollo mayor en la zona de la laguna de decantación donde el material depositado es más fino. En la zona de vertido de las PR estudiadas no son frecuentes las eflorescencias salinas, pero sí se observan en taludes y cortes, en correspondencia con capas de arena (Fig. 5). En las calicatas se pudo observar que algunos de los estratos limosos se encuentran oxidados. La oxidación es intensa hasta los $50 \mathrm{~cm}$ de profundidad y notablemente más leve a profundidades mayores.

Se realizaron cuatro sondeos con pala manual, de $16 \mathrm{~cm}$ de diámetro y 7,00 $\mathrm{m}$ de profundidad en tres sitios de la PR CV1, uno en la zona de la laguna de decantación (S1), dos en la zona transición (S2 y S2-SPT) y el restante en la zona de vertido (S3). Además, se llevó a cabo un quinto (S4) sondeo de características similares en la PR CV2. La ubicación aproximada de los sondeos se muestra en la fotografía de la Figura 3 y Figura 4.

Cada $25 \mathrm{~cm}$ de avance de la perforación se recuperaron muestras alteradas. A partir de las muestras recuperadas se dibujaron los logs de sondeo (Fig. 6) y se determinó en laboratorio el perfil vertical de humedad gravimétrica. En los niveles marcados con " $\mathrm{G}$ " se obtuvieron muestras para granulometría.

Comparando los materiales identificados en los lodos de los sondeos S1, S2 y S3, se aprecia cómo, en promedio, el tamaño de las partículas disminuye des-

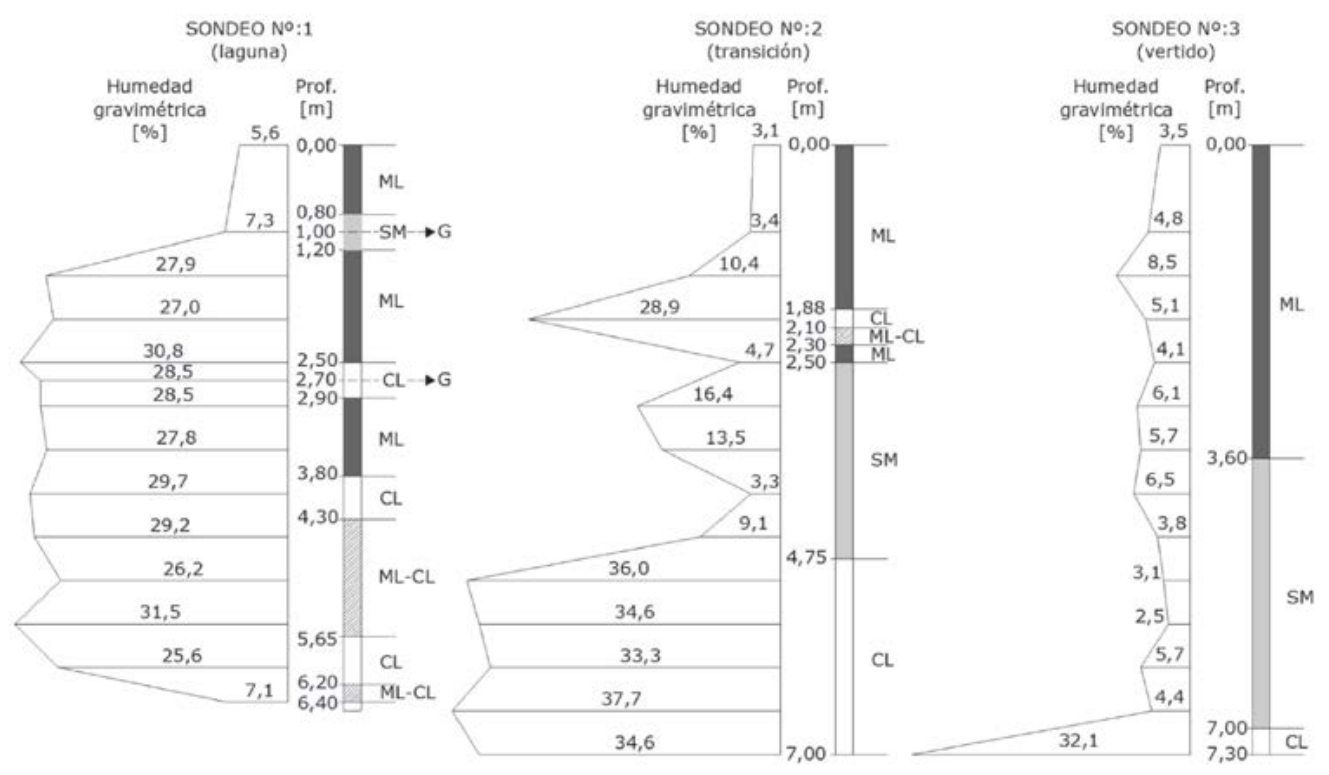

Figura 6. Estratigrafía y perfil vertical de humedad a partir del material recuperado en las perforaciones manuales en la PR CV1. G: punto de toma de muestras para granulometría.

Figure 6. Stratigraphic and gravimetric water content profile from the material recovered in the manual boreholes in the CV1 tailings dam. G: grain size distribution samples. 
de la zona de vertido hacia la zona de la laguna. Todos los sondeos alcanzaron profundidades cercanas a los $7 \mathrm{~m}$, sin encontrar un nivel freático en ningún caso. Tampoco se observaron en las inmediaciones de la PR vertientes naturales ni escorrentía superficial permanente. Se encuentran algunos pequeños cauces temporales de escorrentía de agua de Iluvia. En general la vegetación es escasa y adaptada al déficit hídrico.

En contraste con el paisaje árido, los sondeos S1 y S2 muestran que, superada una capa superficial de 1 metro y 1.5 metros de espesor, respectivamente, se encuentran relaves con humedades sorprendentemente elevadas. Una estimación de los grados de saturación a partir de los datos de humedad y de peso unitario medido en calicatas da valores entre el 80 y el $100 \%$.

Este hallazgo resulta notable si se tiene en cuenta que estos relaves han permanecido más de 50 años interactuando libremente con la atmósfera en un clima árido. El déficit hídrico está presente todos los meses del año (no hay estación húmeda) y el déficit medio supera los $1000 \mathrm{~mm}$ anuales. Grados de saturación elevados también han sido reportados en las PR simi- lares ubicadas en la Sierra de Cartagena-La Unión, en la Región de Murcia, España, pero bajo condiciones atmosféricas no tan severas (Oldecop et al., 2011).

El sondeo S3 presenta valores comparativamente bajos de humedad en la mayor parte de su longitud. Un posible motivo de esto es que el sondeo se encuentra próximo al talud frontal de la presa, que constituye una frontera de evaporación. Sin embargo, a partir de los $7,00 \mathrm{~m}$ y hasta los 7,30 $\mathrm{m}$ de profundidad (fin de la excavación) se encontró una arcilla casi saturada.

Llama también la atención que los valores de humedad elevados se encuentran sistemáticamente en los finos, limos y arcillas, con la única excepción de los primeros 3.6 metros de limo del sondeo S3, probablemente por la razón antes indicada. En cambio, donde se encuentran arenas, la humedad cae abruptamente.

El motivo de este comportamiento se comprende mejor con los datos del sondeo S4, realizado en la PR CV2. El log y el perfil de humedad se muestran en la Figura 7. Tal como muestran las curvas granulométricas que se presentan en la Figura 8, los tres tipos de relaves encontrados en la PR CV2 son prácticamente

SONDEO NO:4
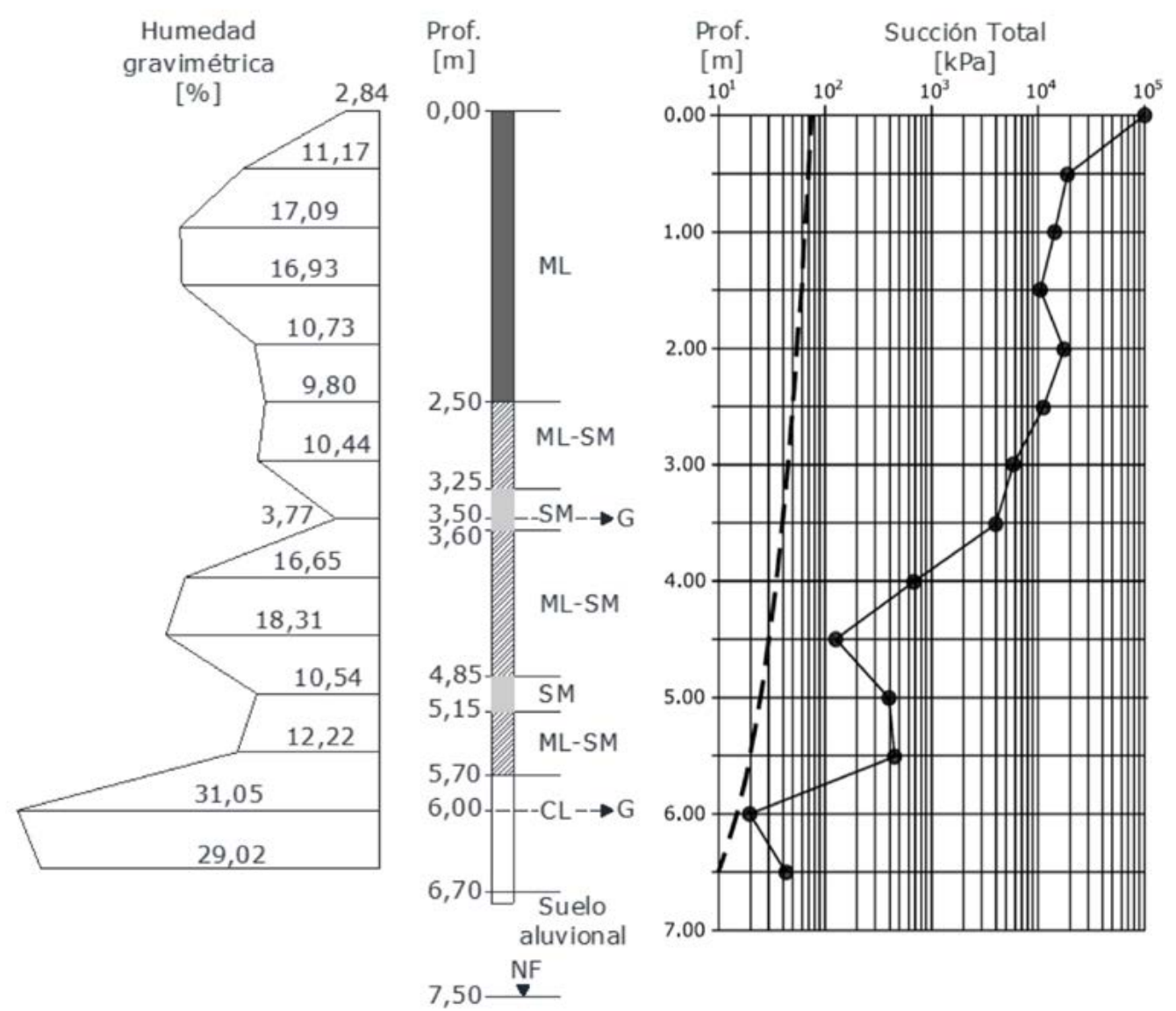

Figura 7. Perfil estratigráfico (centro), de humedad gravimétrica (izquierda) y de succión total (derecha) para muestras recuperadas del sondeo S4, CV2. G: punto de toma de muestras para granulometría.

Figure 7. Stratigraphic profiles (left), degree of saturation (centre) and total suction (right) for samples recovered from S4 borehole, CV2 tailings dam. G: grain size distribution samples. 
idénticos a los de la PR CV1, con independencia de la profundidad de donde se obtuvo la muestra. Además, se determinó para cada muestra la succión total por medio de un psicrómetro de punto de rocío marca Decagon modelo WP4-T. Los resultados se muestran a la derecha de la Figura 7, en donde también se indica como referencia el perfil de presiones hidrostático. La estratigrafía y el perfil de humedad del sondeo S4 resultan similares al del S2, de la zona de transición del CV1. En ambos casos se alternan los limos y arcillas con capas de arena.

Se aprecian valores elevados de succión total, sobre todo en los estratos superficiales, disminuyendo en profundidad. En todo el espesor de relaves la succión está por arriba del perfil hidrostático, aproximándose hacia el fondo del sondeo. Nótese que en este caso existe un nivel freático en el suelo de fundación, debido a la presencia de un curso de agua permanente, vecino al depósito. En general el gradiente de succiones se corresponde con un flujo capilar ascendente inducido por evaporación. La excepción es el gradiente invertido entre los 1.5 y $2 \mathrm{~m}$ de profundidad, que puede ser un relicto de un evento de infiltración de agua de lluvia. En cambio, la oscilación por debajo de los $4.5 \mathrm{~m}$ es muy probable que se deba a la variabilidad del método de medición. En cualquier caso, por debajo de los $4.5 \mathrm{~m}$ de profundidad, la succión es baja y tiende a aproximarse al perfil hidrostático.

En esta condición debe existir equilibrio de presiones (succiones) en la interfaz entre estratos vecinos y por lo tanto los cambios bruscos de humedad son causados por las diferencias entre curvas de retención o curvas características de los diferentes materiales del perfil estratigráfico. En el proceso de secado, los estratos finos mantienen elevados grados de saturación mientras que en los de arena se desaturan quedando sus poros mayoritariamente llenos de aire.

\section{Ensayo de parcela}

En la PR CV1, se realizó un experimento de campo con el objeto de estudiar el rol de la estratificación en la interacción con la atmósfera de los relaves. EI experimento se extendió durante 527 días entre el 23 de octubre de 2012 y el 3 de marzo de 2014.

\section{Preparación del ensayo}

El ensayo consistió en aislar hidráulicamente una parcela de $1,0 \mathrm{~m}$ de lado y $0,8 \mathrm{~m}$ de profundidad, para lo cual se excavo una trinchera, que a la vez permitiera la instalación de los sensores en los laterales del volumen de ensayo. Para evitar el flujo lateral se colocó una barrera de polietileno rodeando la parcela (Fig. 9).

Distribuidos en las cuatro caras de la parcela, se instalaron los sensores de humedad volumétrica marca Decagon, modelos 5TM y EC-5 y de succión matricial marca Decagon, modelo MPS-2, (Tabla 1). Su principio de funcionamiento se basa en la medición de la

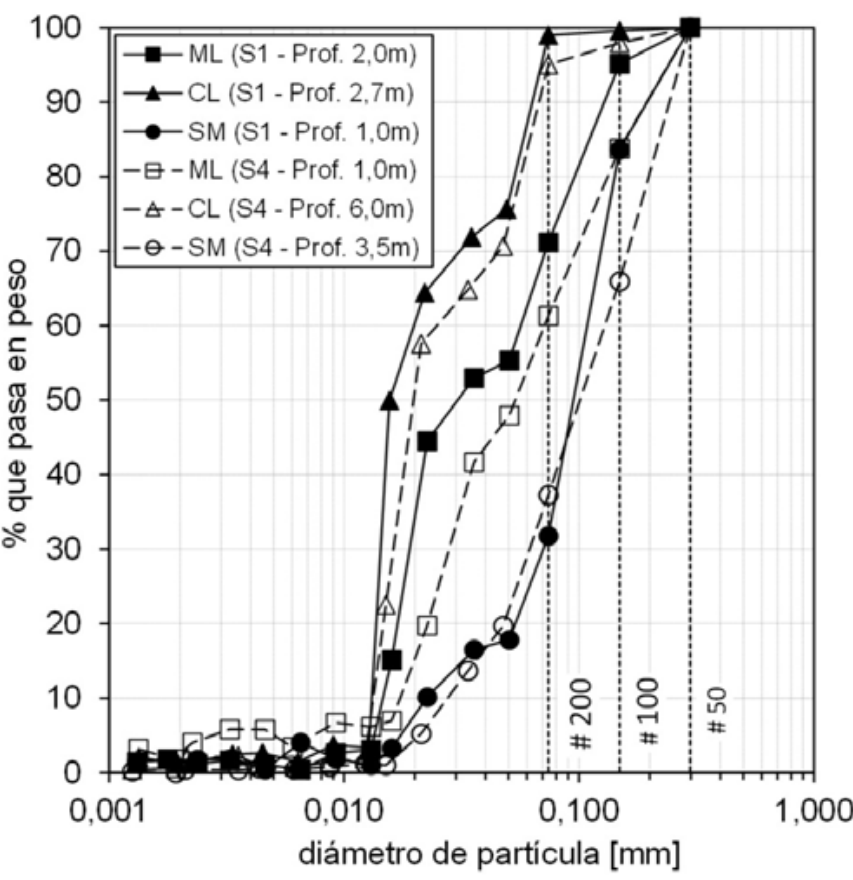

Figura 8. Curvas granulométricas para muestras de los sondeos S1 y S4.

Figure 8. Grain size distribution curves for samples of the $S 1$ and S4 boreholes.

permitividad dieléctrica. Adicionalmente, todos los sensores registran la temperatura del suelo. La Figura 10 muestra un perfil simplificado de la estratigrafía, en donde se indica la posición de los sensores.

\section{Fases del ensayo}

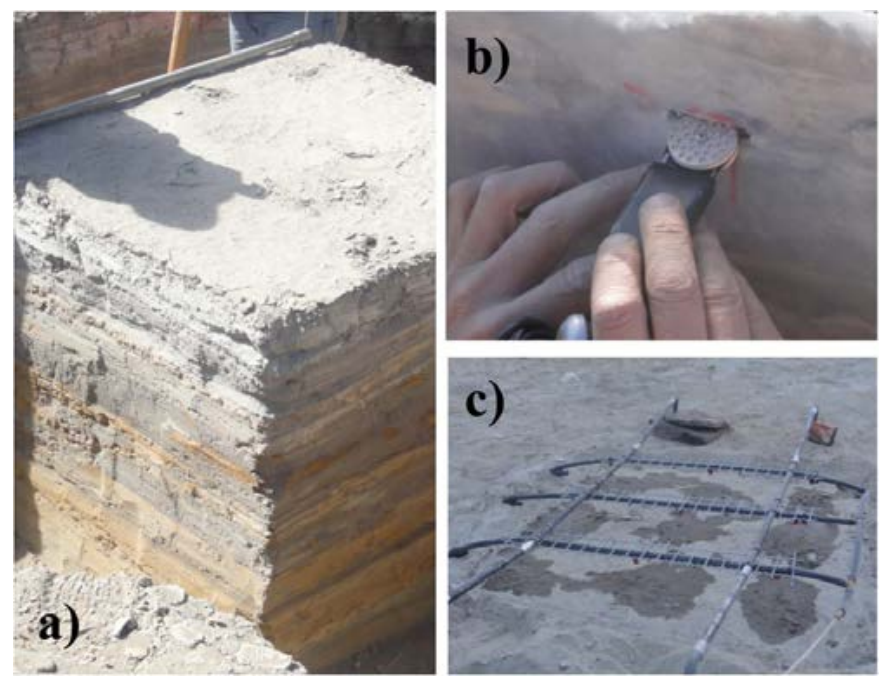

Figura 9. a) Perfil donde se aprecia la estratigrafía de la parcela de ensayo, nótese la variación del color debido a los diferentes grados de oxidación. b) Instalación de sensores a través de la barrera impermeable. c) Sistema de riego.

Figure 9. a) Stratigraphy of the test plot. b) Sensor installation through the impermeable barrier. c) Irrigation system. 


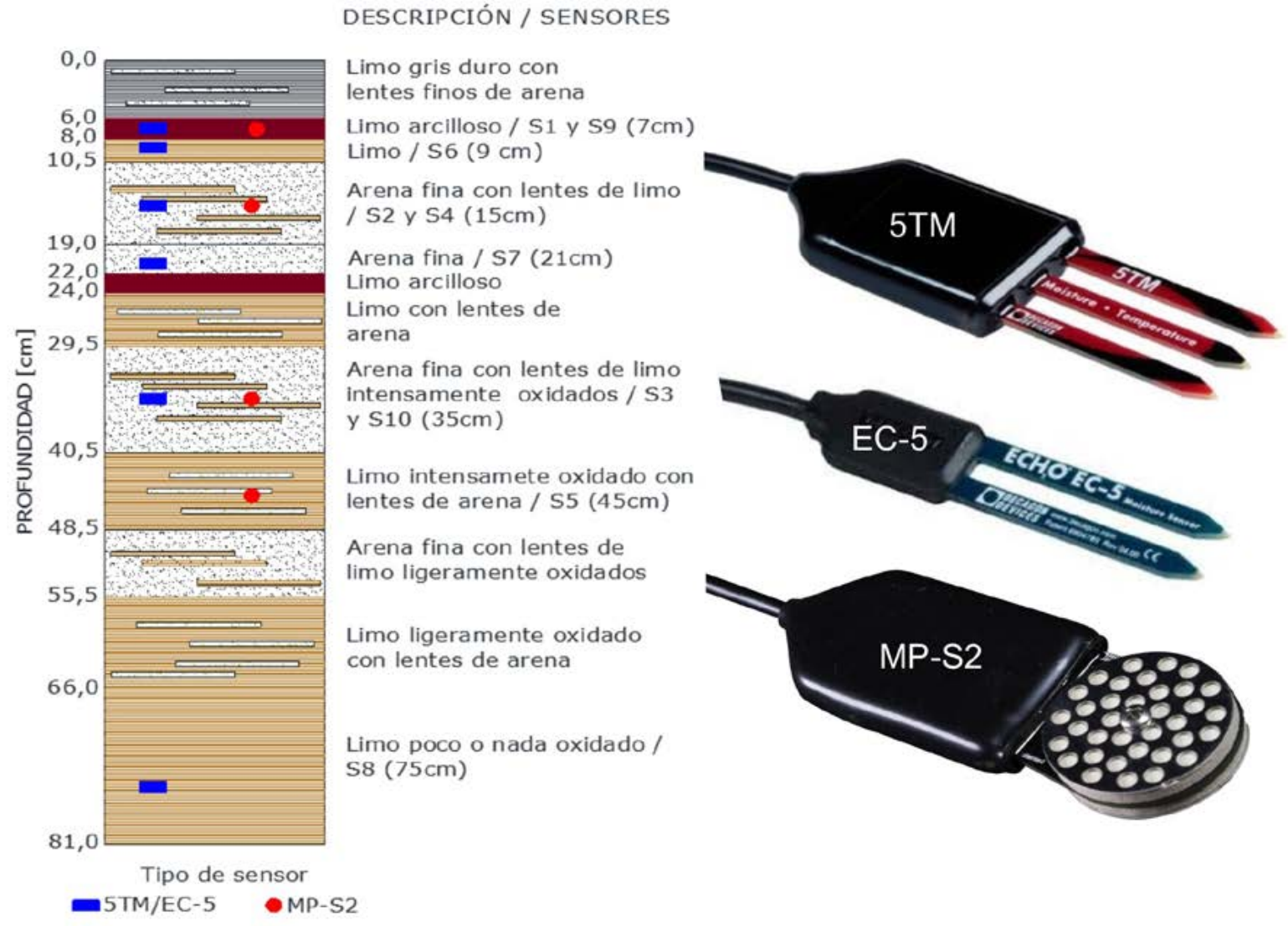

Figura 10. Perfil estratigráfico simplificado de la parcela estudiada y ubicación de los sensores.

Figure 10. Simplified stratigraphic profile and height location of the sensors.

\begin{tabular}{ccccc}
\hline Denominación & Modelo & Posición & Profundidad [cm] (material) & Variables \\
\hline S1 & 5TM & Oeste & 7 (Limo arcilloso) & $\mathbf{A}_{\mathrm{w}} \mathrm{y} \mathrm{T}$ \\
S2 & 5TM & Oeste & 15 (Arena fina) & $\mathbf{A}_{\mathrm{w}} \mathrm{y} \mathrm{T}$ \\
S3 & EC-5 & Oeste & 35 (Arena fina) & $\mathbf{A}_{\mathrm{w}} \mathrm{y} \mathrm{T}$ \\
S4 & MPS-2 & Norte & 15 (Arena fina) & $\mathrm{s}$ \\
S5 & MPS-2 & Norte & 45 (Limo arenosos) & $\mathrm{s}$ \\
S6 & 5TM & Este & 9 (Limo) & $\mathbf{A}_{\mathrm{w}} \mathrm{y} \mathrm{T}$ \\
S7 & 5TM & Este & 21 (Arena fina) & $\mathbf{A}_{\mathrm{w}} \mathrm{y} \mathrm{T}$ \\
S8 & EC-5 & Este & 75 (Limo arenoso) & $\mathbf{A}_{\mathrm{w}} \mathrm{y} \mathrm{T}$ \\
S9 & MPS-2 & Sur & 7 (Limo arcilloso) & $\mathrm{s}$ \\
S10 & MPS-2 & Sur & 35 (Arena fina) & $\mathrm{s}$ \\
\hline
\end{tabular}

$\mathbf{A}_{\mathrm{w}}=$ contenido volumétrico de agua, $\mathrm{T}=$ =temperatura y s=succión matricial

Tabla 1. Características y posición de los sensores utilizados.

Table 1. Characteristics and position of the installed sensors. 
El experimento se dividió en dos fases. En la Fase A (inicio el 23/10/2012) se efectuaron aportes de agua mediante un sistema de riego por aspersión, durante tres días. En esta fase se aportaron 122,25 I distribuidos en 12 riegos (Figura 11). En todos los riegos la tasa utilizada fue de $20 \mathrm{~mm} /$ hora, pero la duración y espaciado de los riegos se hizo de tal manera que no se generara encharcamiento ni escurrimiento superficial. Simultáneamente se comenzaron a registrar las lecturas de los sensores, que se convirtieron a valores de humedad gravimétrica a partir de determinaciones de peso unitario y densidad de sólidos hechas sobre muestras tomadas en las calicatas. En la Tabla 2 se describen los riegos artificiales realizados en esta fase de ensayo.

Una vez concluida la primera fase de ensayo, se permitió que la superficie de la parcela interactuara

\begin{tabular}{cccc}
\hline Riego & $\begin{array}{c}\mathrm{T} \\
{[\text { horas }]}\end{array}$ & $\begin{array}{c}\text { Dt } \\
{[\mathrm{min}]}\end{array}$ & $\begin{array}{c}\text { Vol } \\
{[\mathrm{lts}]}\end{array}$ \\
\hline 1 & $1,17-1,75$ & 34,8 & 12,00 \\
2 & $3,17-3,83$ & 39,6 & 13,21 \\
3 & $4,33-5,00$ & 40,2 & 11,99 \\
4 & $5,33-6,00$ & 40,2 & 13,63 \\
5 & $9,58-10,17$ & 41,4 & 12,18 \\
6 & $10,58-11,17$ & 35,4 & 12,23 \\
7 & $11,58-11,83$ & 15,0 & 5,12 \\
8 & $13,42-13,67$ & 15,0 & 4,75 \\
9 & $46,25-46,83$ & 34,8 & 11,84 \\
10 & $47,42-47,83$ & 24,6 & 8,85 \\
11 & $62,32-62,92$ & 36,0 & 11,12 \\
12 & $64,83-65,25$ & 25,2 & 8,30 \\
\hline
\end{tabular}

Tabla 2. Secuencia de riegos realizados durante la Fase A. Table 2. Sequence of irrigations carried out during Phase $A$.

\begin{tabular}{|c|c|c|c|c|c|}
\hline \multicolumn{3}{|c|}{ Año 2013} & \multicolumn{3}{|c|}{ Año 2014} \\
\hline Fecha & $\begin{array}{c}\text { Duración } \\
\text { [min] }\end{array}$ & $\begin{array}{c}\mathrm{P} \\
{[\mathrm{mm}]}\end{array}$ & Fecha & $\begin{array}{l}\text { Duración } \\
\text { [minutos] }\end{array}$ & $\begin{array}{c}\mathrm{P} \\
{[\mathrm{mm}]}\end{array}$ \\
\hline $15 / 01 / 2013$ & 45 & 14 & $14 / 01 / 2014$ & 30 & 7 \\
\hline $27 / 01 / 2013$ & 30 & 7 & $12 / 02 / 2014$ & 15 & 7 \\
\hline $05 / 02 / 2013$ & 75 & 20 & $14 / 02 / 2014$ & 120 & 10 \\
\hline $07 / 02 / 2013$ & 90 & 21 & $18 / 02 / 2014$ & 45 & 8 \\
\hline $07 / 02 / 2013$ & 30 & 6 & - & - & 0 \\
\hline $01 / 11 / 2013$ & 120 & 11 & - & - & 0 \\
\hline \multicolumn{2}{|c|}{ Total lluvias intensas } & 79 & \multicolumn{2}{|c|}{ Total lluvias intensas } & 32 \\
\hline \multicolumn{2}{|c|}{ Total lluvias dispersas } & 64 & \multicolumn{2}{|c|}{ Total lluvias dispersas } & 61 \\
\hline \multicolumn{2}{|c|}{ Precipitación total } & 143 & \multicolumn{2}{|c|}{ Precipitación total } & 93 \\
\hline
\end{tabular}

Tabla 3. Lluvias torrenciales y precipitación total (registro 23/10/2012 a 4/4/2014).

Table 3. Torrential rain and total precipitation (register 23/10/2012 to $4 / 4 / 2014$ ). libremente con la atmósfera por más de un año y medio. Estas es la denominada Fase B del ensayo (entre el 26/10/2012 y el 04/04/2014). Se instaló además una estación meteorológica en las proximidades de la PR CV1, donde se registró la temperatura ambiente, la humedad relativa y las precipitaciones durante todo el periodo de ensayo. En la Tabla 3 se muestran las Iluvias torrenciales y la precipitación total registradas durante la fase $B$.

\section{Análisis de los registros}

\section{Variación de la humedad}

La variación de la humedad gravimétrica obtenida a partir de los registros de los sensores durante la Fase A se muestra superpuesta con los aportes de agua efectuados en el mismo periodo de tiempo (Fig. 11). El volumen de agua aplicado equivale a una precipitación de $125 \mathrm{~mm}$ en 3 días. Esto constituiría un evento totalmente improbable en el sitio de estudio, considerando la aridez del clima y los registros meteorológicos históricos. Los dos sensores superficiales (S1 y S6) reaccionaron con un retraso de 14 hs respecto del inicio de los riegos (recarga artificial). La capa superficial donde estaba instalado el sensor S1 está formada por estratos delgados y extremadamente duros que presentan un patrón de agrietamiento no muy evidente hasta que se excava (Fig. 5).

El sensor $\mathrm{S} 6$ ubicado a $9 \mathrm{~cm}$ de profundidad reaccionó antes que el sensor S1 ubicado a $7 \mathrm{~cm}$ de profundidad aunque a partir de las 46 horas el comportamiento de ambos resulta similar. Esto puede estar relacionado con el agrietamiento de la capa superficial que provoca un flujo preferencial, luego detenido por la capa de limo más uniforme e impermeable que la anterior, donde estaba instalado el sensor S6.

Los sensores más profundos (S2, S3, S7 y S8), se mantuvieron sin reaccionar y en valores muy bajos de humedad $(<2 \%)$ durante los riegos 1 a 8 . Luego, sorpresivamente, el sensor $\mathrm{S} 8$, ubicado a $75 \mathrm{~cm}$, registró un aumento brusco de la humedad 18 horas después del inicio del ensayo. Se puede pensar que incluso replica el comportamiento del S6, mucho más superficial. Por ejemplo, la pausa de 6 horas entre los riegos 4 y 5 , se observa en ambos sensores, con los esperables retrasos. Sin embargo, con posterioridad a la pausa de 32 horas entre los riegos 8 y 9 , el sensor $S 8$ no volvió a reaccionar a ningún evento de infiltración posterior, en tanto que el S6 lo siguió haciendo. Los sensores S2, S3 y S7 (Prof. = 15, 35 y $21 \mathrm{~cm}$ respectivamente) reaccionaron recién 2 días y medio luego de iniciado el ensayo, coincidiendo con los dos últimos riegos aplicados (11 y 12) y además lo hicieron de manera mucho más lenta que el resto.

Es interesante notar que los tres sensores instalados en estratos de limo ( $\mathrm{S} 1, \mathrm{~S} 6$ y $\mathrm{S} 8$ ) reaccionaron antes y de forma más acusada que los tres sensores 


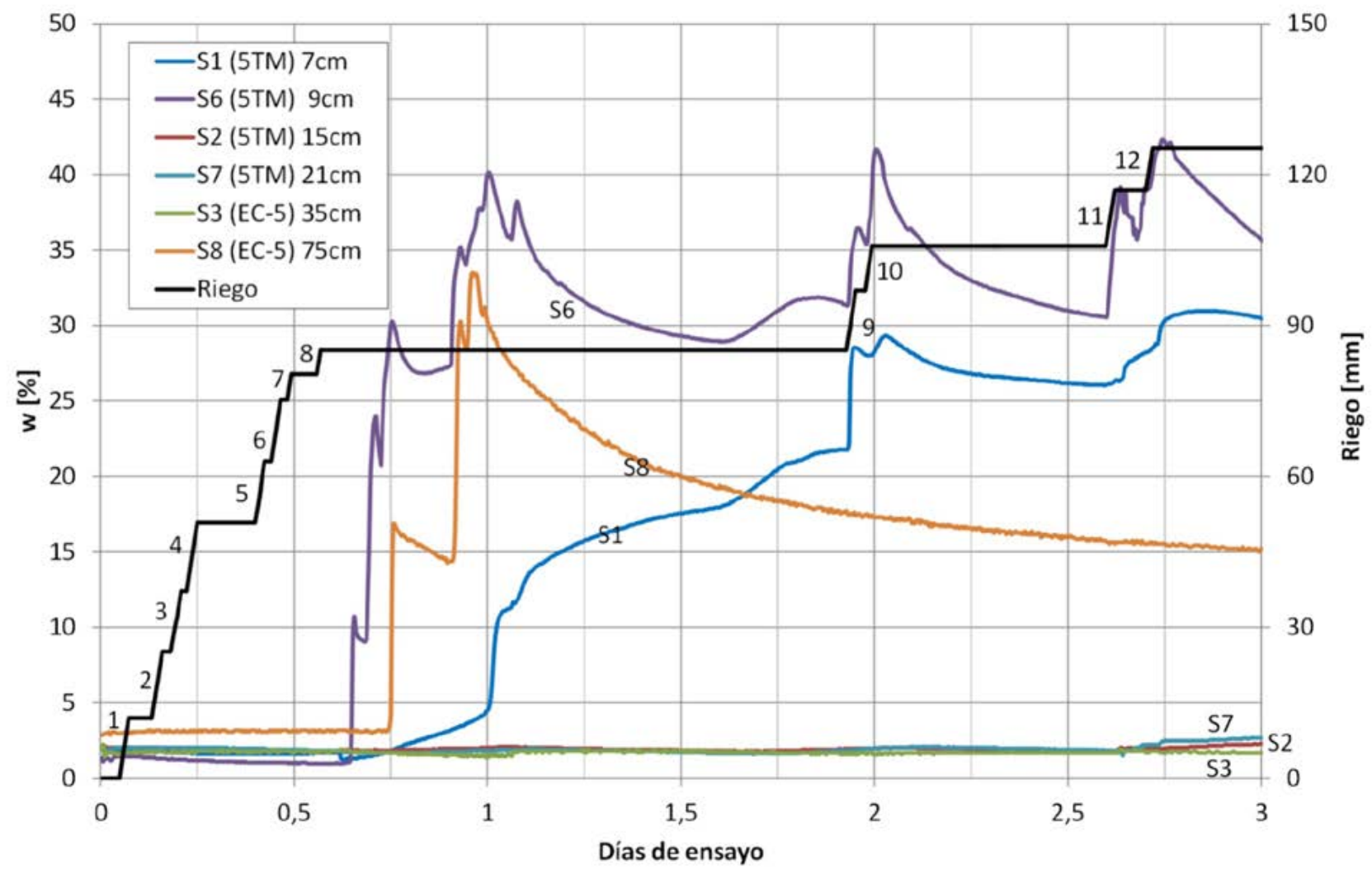

Figura 11. Variación de la humedad gravimétrica durante la Fase $A$. Figure 11. Variation of water content during Phase A.

instalados en estratos arena (S2, S3 y S7), sin que el orden de su respuesta tenga una correlación clara con la profundidad de instalación.

La Figura 12 muestra los 4 primeros meses de ensayo. Se puede observar la fluctuación en las mediciones causadas por las variaciones de temperatura, que afectan más a los sensores superficiales que a los profundos. Esto es un artefacto del tipo de sensor utilizado (Dorigo et al., 2013; Kapilaratne and Lu, 2017), que aquí no se ha intentado corregir.

Finalizada la Fase A, los sensores $\mathrm{S} 1, \mathrm{~S} 6$ y $\mathrm{S} 8$ (en limo), comenzaron a registrar un descenso sostenido de la humedad, alcanzando valores comprendidos entre el 15 y $25 \%$ (Sr entre 20 y $60 \%$ respectivamente), al cabo de un mes. En contraste, los sensores en arena S2, S3 y S7 incrementaron lenta y sostenidamente sus lecturas hasta el día 15 en el caso del S7, hasta el día 30 el S2 y hasta el día 60 el S3.

Pasados los 60 días de ensayo sin nuevos aportes de agua, todos los sensores, excepto el S7, se estabilizaron en valores de humedad relativamente bajos, entre 3 y $6 \%$ ( $\mathrm{Sr}$ entre 15 y 30\% respectivamente), aunque mayores a la humedad medida inicialmente. Por el contrario, el sensor S7 se estabilizó en una lectura cercana $20 \%$ ( $\mathrm{Sr} 50 \%$ ). Es posible que este sensor, por proximidad, estuviera midiendo la humead de la capa vecina de arcilla o bien una media entre la arcilla y la arena (el volumen de medición del sensor equivale a un cilindro de aproximadamente $10 \mathrm{~cm}$ de diámetro).

La Figura 13 muestra los registros de humedad de todo el ensayo. A partir de mediados de enero hasta mediados de febrero de 2013, ocurrieron las primeras lluvias desde el inicio del ensayo (Figura 13). Estas fueron de tipo torrencial, típicas del verano. Los sensores superficiales (S1 y S6), que se encontraban con humedades inferiores al 5\%, fueron los primeros en reaccionar, seguidos del sensor S7 y S2, este último con un valor de humedad menor a pesar de ser más superficial. Por último, reacciona el sensor S3, con casi 10 días de retraso respecto del inicio de las lluvias y además lo hace muy suavemente. El sensor S8 no muestra ninguna alteración (Fig. 13). A diferencia del ensayo de riego, se observa que orden temporal de reacción de los sensores, es consistente con un frente de infiltración.

A finales de mayo de 2013 (día de ensayo $N^{\circ} 216$ ) ocurrió un nuevo episodio de lluvia. Es un evento típico de otoño-invierno, de magnitud muy inferior a las lluvias de verano $(P=11 \mathrm{~mm})$ y además aislado. Provocó en los sensores superficiales S1 y S6 una reacción muy rápida en tanto que en todos los demás sensores no hay una reacción evidente.

En los 6 meses finales, algunos sensores como el S2 dejan de medir (día N³65) y otros, como el S3, comienzan a tener lecturas 


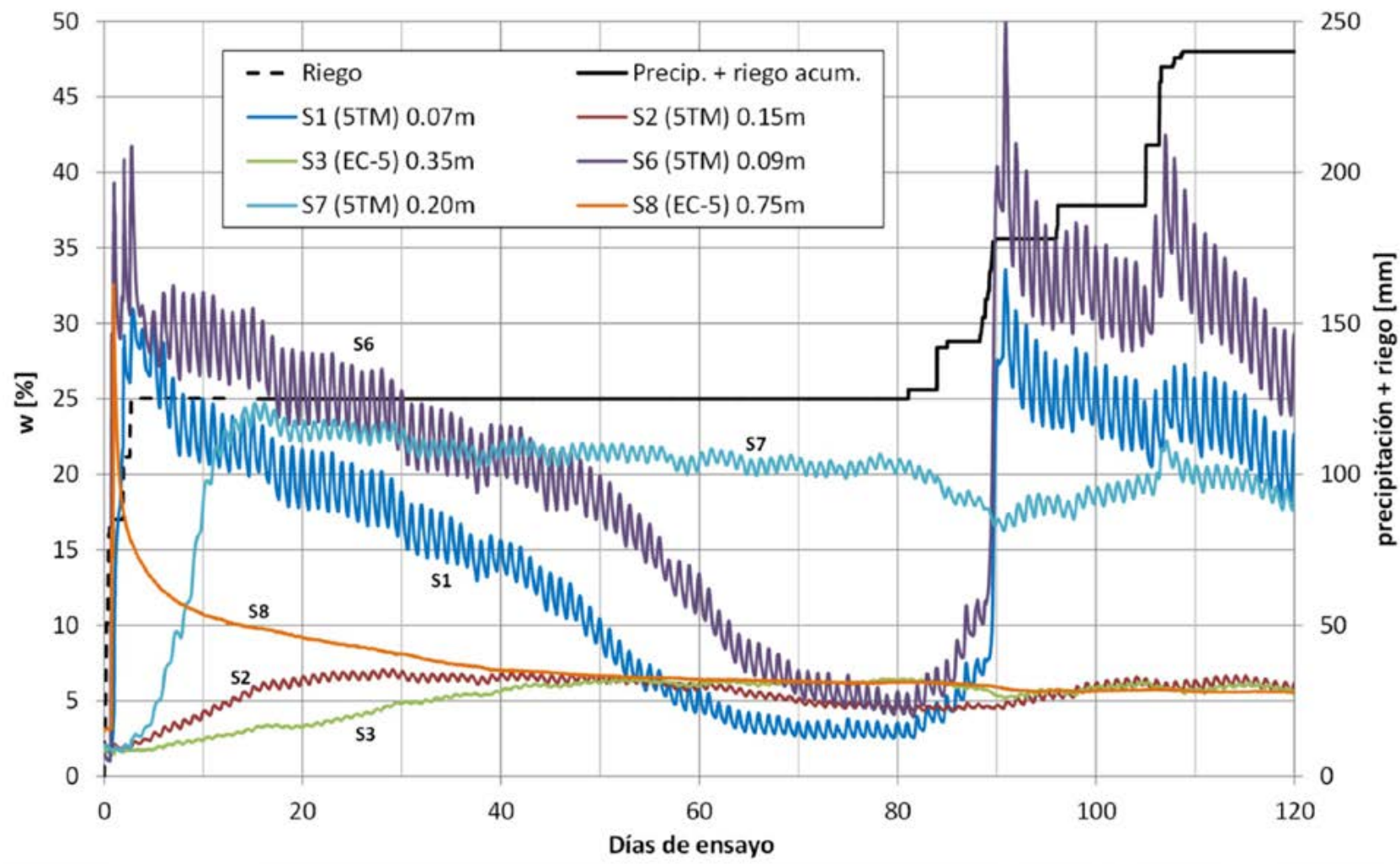

Figura 12. Variación de la humedad gravimétrica durante los 6 primeros meses de ensayo.

Figure 12. Variation of water content during the first 6 month of the experiment.

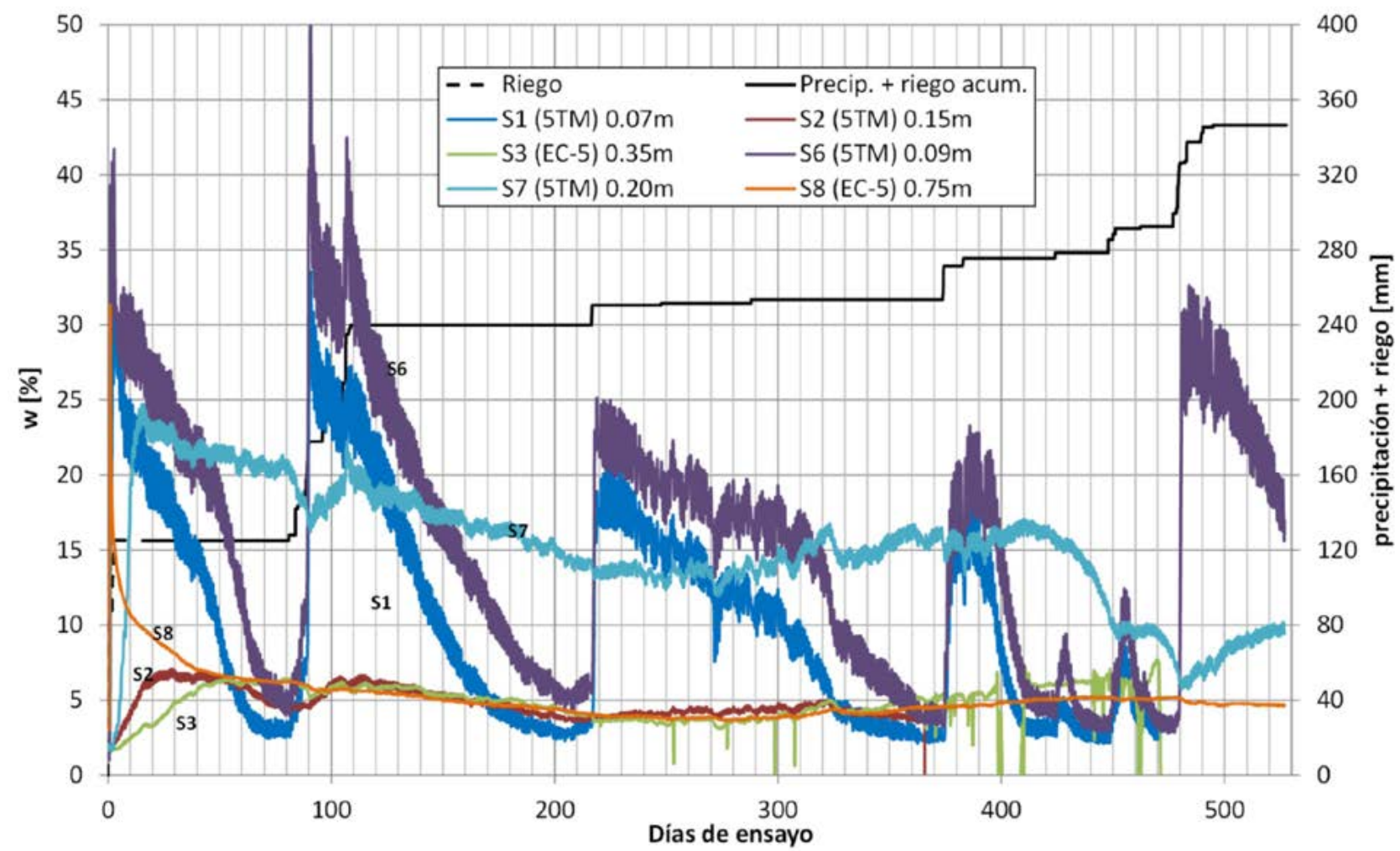

Figura 13. Variación de la humedad gravimétrica durante todo el ensayo.

Figure 13. Variation of water content during the whole test. 
erráticas. El registrador de datos al que estaban conectados los sensores S1, S2 y S3 deja de adquirir datos el 7 de febrero de 2014, transcurridos 471 días de ensayo. El segundo registrador (S6, S7 y S8) continúa en funcionamiento hasta la fecha de desmantelamiento (3/4/2014).

Las Iluvias del verano 2013-2014 comenzaron a final de octubre (día 373). Sin embargo, después de un primer evento de $18 \mathrm{~mm}$, las lluvias que siguieron en los tres meses siguientes fueron de magnitud reducida, $7 \mathrm{~mm}$ el mayor de ellos en enero (día 448). Recién a partir de mediados de febrero de 2014 ocurrió una sucesión de lluvias que aportaron $53 \mathrm{~mm}$ en 13 días. En todos los eventos, grandes o pequeños, los sensores superficiales S1 y S6 continuaron reaccionando rápidamente y con amplitudes que guardan relación con la magnitud de las lluvias que los provocan. El sensor S7 también muestra una reacción a los eventos de lluvia, aunque más retardada y suavizada que la de los sensores superficiales y además superpuesta con una tendencia de secado entre los días 420 a 480 (mitad de diciembre a mitad de febrero). Esto puede tener relación con la combinación de altas temperaturas de verano y la relativamente es- casa precipitación en el mismo periodo (comparada con la del primer verano). Sin embargo, a partir de las Iluvias de febrero, el sensor S7 vuelve a incrementar sus lecturas, en tanto que el S6 $(9 \mathrm{~cm})$ reacciona rápidamente para luego mostrar un proceso de secado una vez pasadas las lluvias.

\section{Variación del grado de saturación}

La Figura 14 muestra perfiles de grado de saturación para diferentes tiempos a partir del inicio del ensayo y hasta el día $\mathrm{N}^{\circ} 80$, periodo en el cual no se registran lluvias, por lo que los aportes de agua son exclusivamente los realizados artificialmente durante los primeros 3 días de ensayo $(122,25 \mathrm{~mm})$.

En la Figura 15 los perfiles de grado de saturación corresponden a tiempos comprendidos entre los 80 y 365 días de ensayo (izquierda) y entre los 365 y 427 días de ensayo (derecha). En general, se aprecia el carácter acotado de la capa activa, más aun si se tiene en cuenta que las lluvias aportaron $236 \mathrm{~mm}$ durante la fase B de ensayo.

Variación de la temperatura

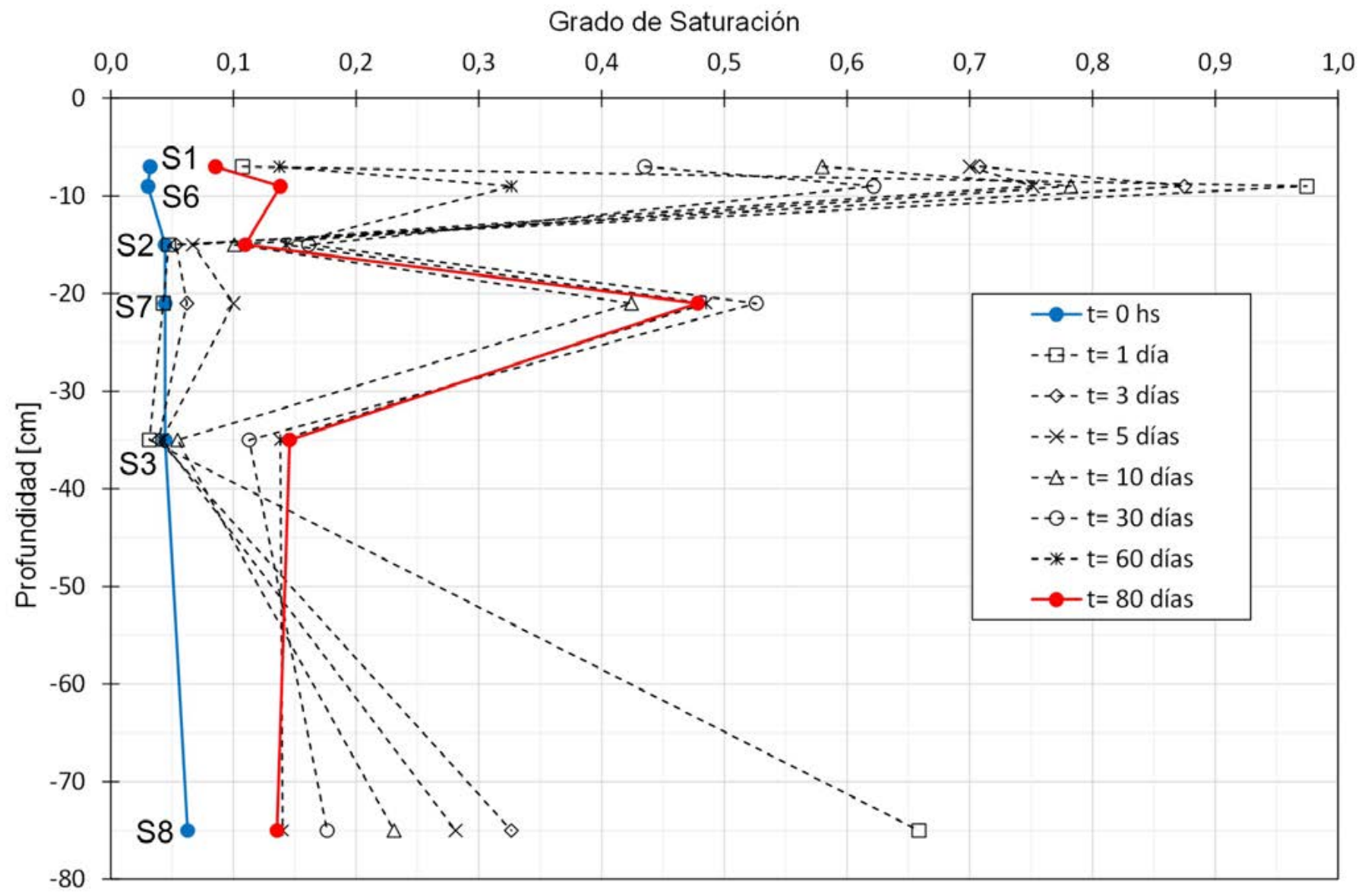

Figura 14. Perfiles de grado de saturación para el intervalo entre 0 y 80 días.

Figure 14. Saturation degree profiles (interval between 0 and 80 days). 


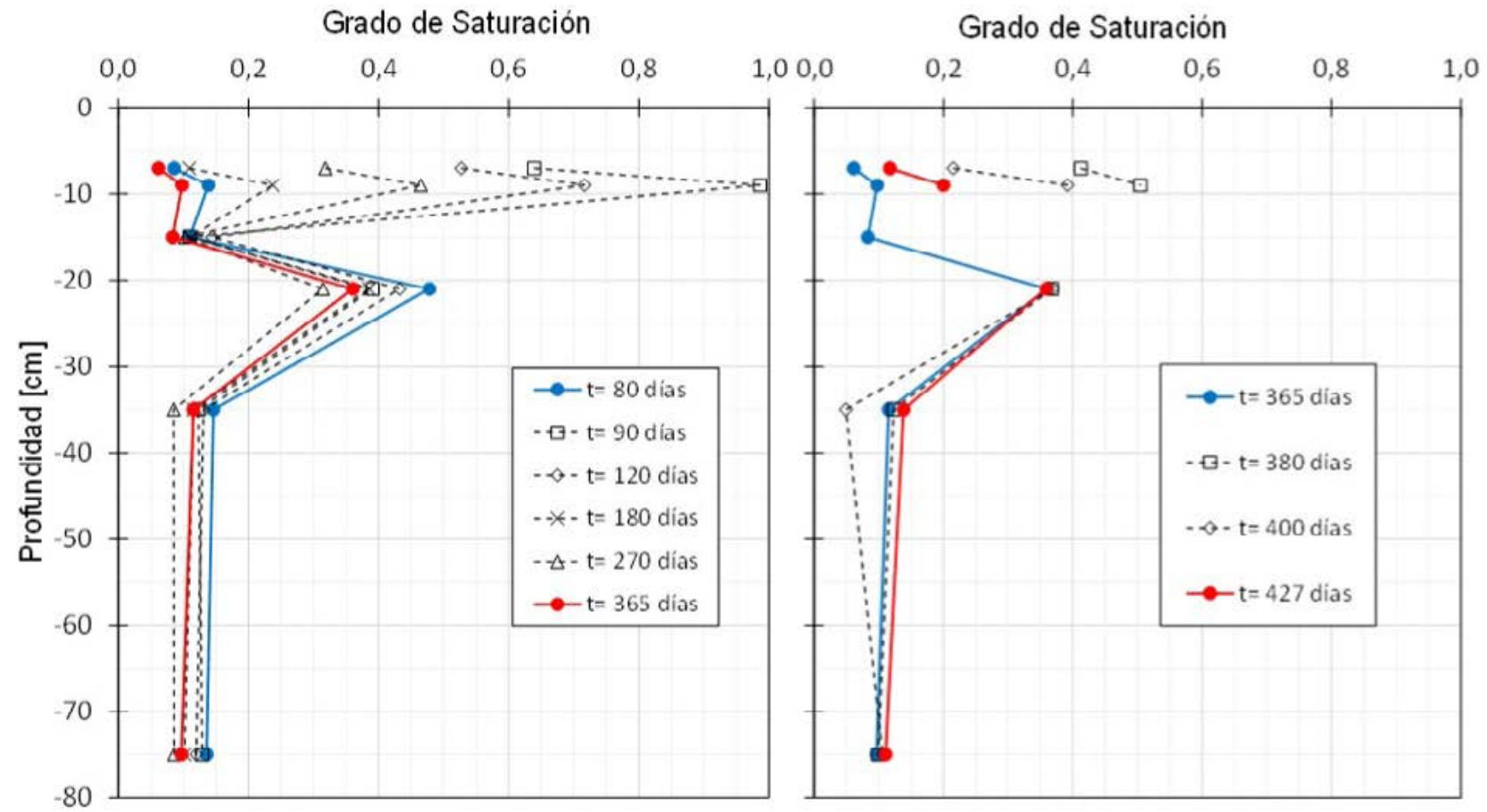

Figura 15. Perfiles de grado de saturación. Intervalo entre 80 días y 365 días (izquierda) y entre 365 y 427 días (derecha). Figure 15. Saturation degree profiles. Interval between 80 days and 365 days (left) and between 365 and 427 days (right).

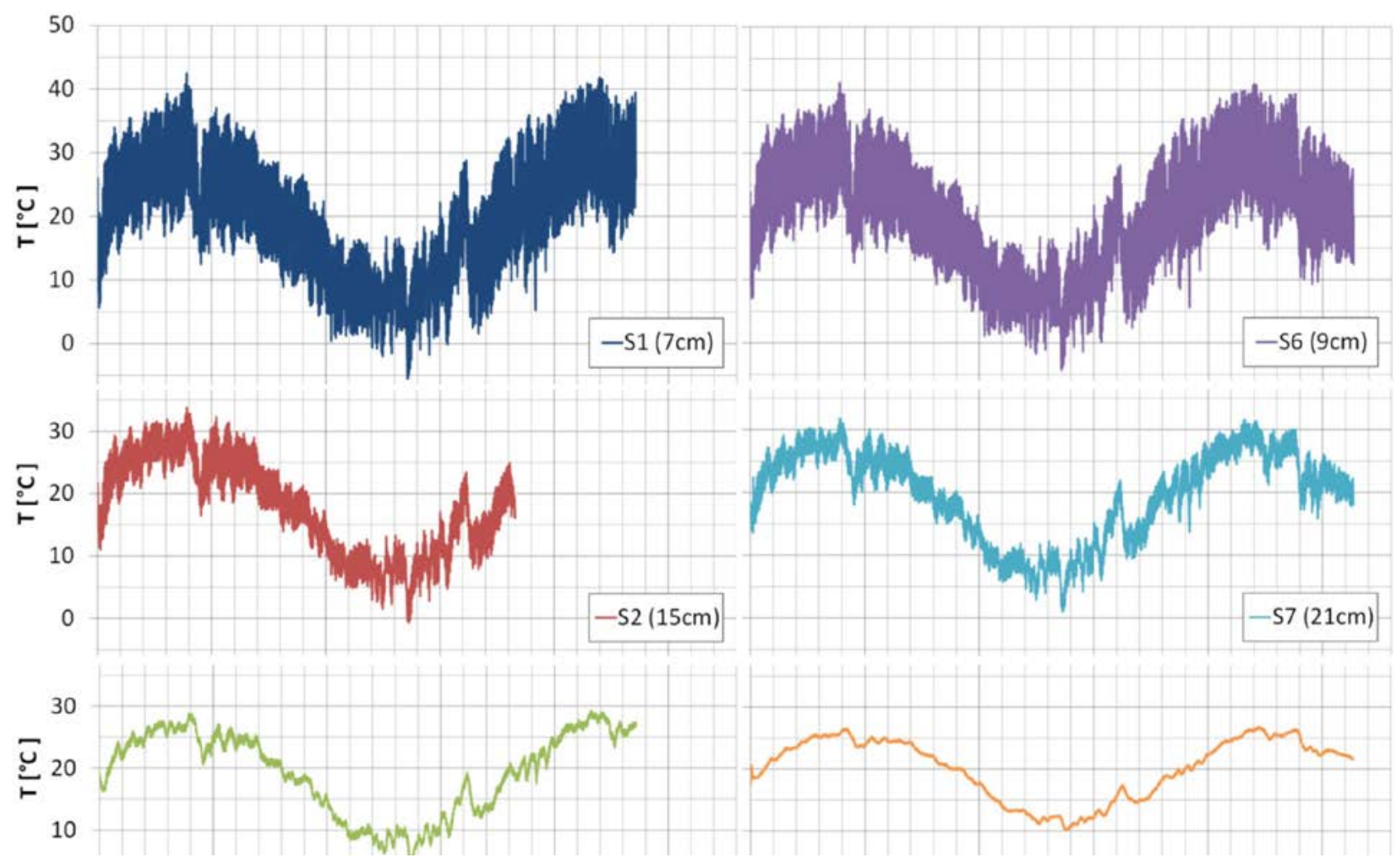

Figura 16. Registros de temperatura a lo largo de todo el ensayo.

Figure 16. Temperature records throughout the whole test. 
La Figura 16 muestra las variaciones de temperatura medidas a diferentes profundidades, para todo el ensayo. Los sensores superficiales registraron en verano temperaturas mayores a los $40^{\circ} \mathrm{C}$ (S1 y S6 ubicados a 7 y $9 \mathrm{~cm}$ respectivamente), mientras que en invierno fueron registradas temperaturas por debajo de cero grados hasta los $35 \mathrm{~cm}$ de profundidad (S2). La amplitud de temperaturas registradas disminuye con la profundidad. Para el sensor más profundo (S8 ubicado a $75 \mathrm{~cm}$ ) las temperaturas máximas registradas son de $25^{\circ} \mathrm{C}$ mientras que las mínimas se encuentran en torno a los $10^{\circ} \mathrm{C}$. Si bien las temperaturas mínimas en los sensores intermedios (S7 y S3) no están por debajo de cero, en el invierno de 2014, llegan a tener temperaturas muy bajas, 1 y 4 grados sobre cero respectivamente

\section{Variación de la succión matricial}

Durante los primeros 60 días no se obtuvieron mediciones de succión debido a un inconveniente con el sistema de adquisición de datos. Por ello sólo se cuenta con medidas de succión en la fase B del ensayo. Los registros de los sensores de succión resultan difíciles de interpretar debido a la fuerte dependencia de las lecturas con la temperatura, que causa fluctuaciones diarias de gran amplitud. La temperatura puede influir de múltiples maneras en las propieda- des dieléctricas (Malmberg and Maryott, 1956; Or and Wraith, 1999; Seyfried and Grant, 2007). Para intentar superar este inconveniente, los datos se corrigieron con la ecuación propuesta por Walthert and Schleppi (2018) para el sensor MPS-2:

$$
\begin{aligned}
& \Delta p F=\left(6.206 \Delta T+0.1137 \Delta T^{2}\right) \times e^{-22.76 /(p F+1.25)} \text { dónde }: \\
& p F=\log
\end{aligned}
$$

(succión $[\mathrm{kPa}]$ ) y $\Delta \mathrm{T}\left[{ }^{\circ} \mathrm{C}\right]$ es la diferencia entre la temperatura actual y la de referencia $\left(22^{\circ} \mathrm{C}\right)$.

La ec. (1) da los valores de succión corregidos a una temperatura de referencia de $22^{\circ} \mathrm{C}$ (temperatura utilizada para la calibración de fábrica) en función de la temperatura y la succión medida. En el proceso de corrección es necesario descartar datos cuando el sensor ha saturado (succión medida $>100 \mathrm{MPa}$ ) o bien cuando las temperaturas estuvieron fuera del rango que se utilizó para el ajuste de la ecuación $\left(4\right.$ a $26^{\circ} \mathrm{C}$ ).

La Figura 17 muestra los registros de succión corregidos. Los datos deben interpretarse con cautela, ya que las correcciones aplicadas van de $-95 \%$ al $+70 \%$ respecto de los valores medidos.

Analizando los registros de succión, se puede ver que el sensor más superficial, S9 $(9 \mathrm{~cm})$, reacciona con una caída brusca de succión ante cada evento de

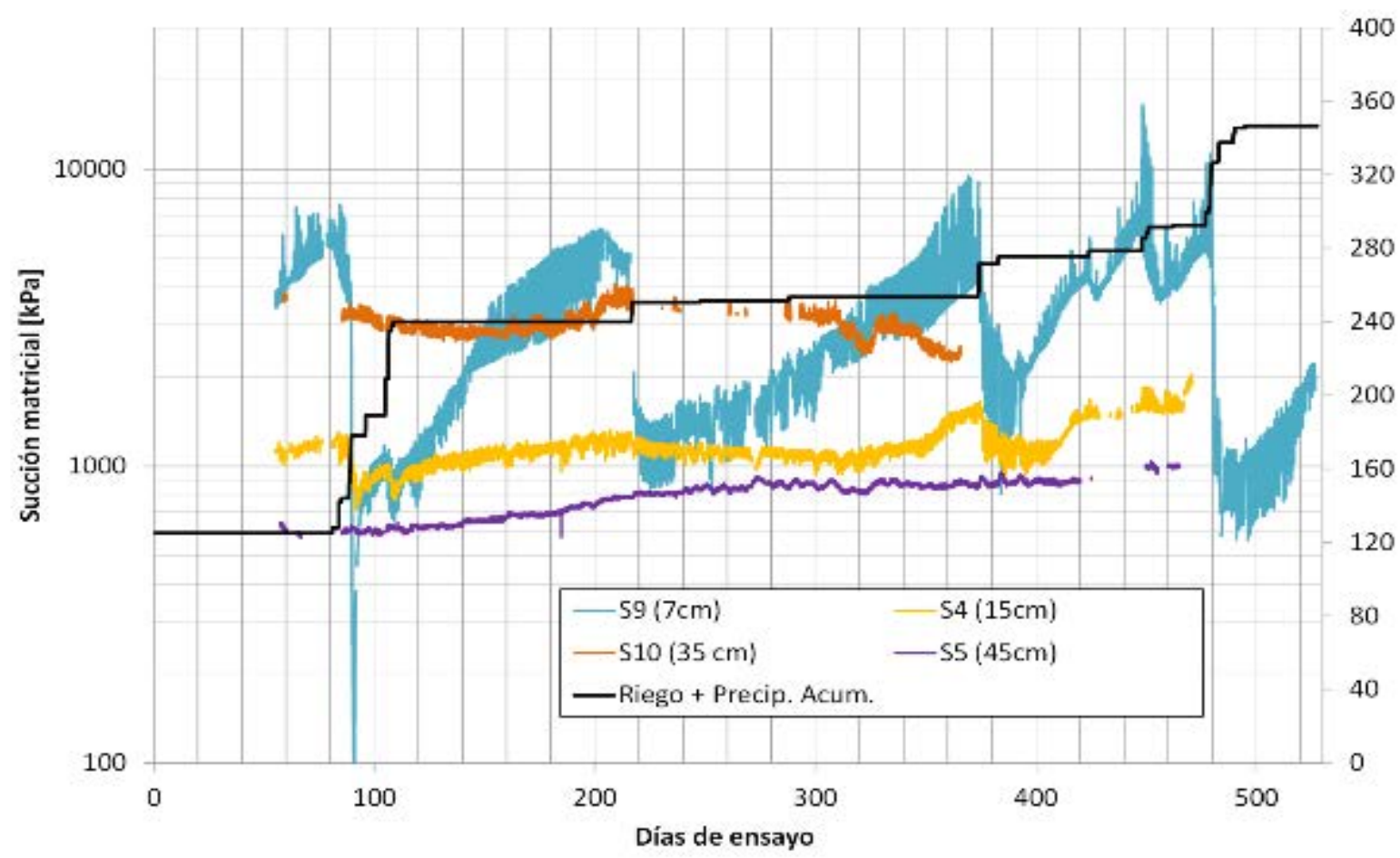

Figura 17. Registros de succión a lo largo de todo el ensayo, corregidos por temperatura. Para referencia se muestra también el registro de riego + lluvia acumulada.

Figure 17. Temperature-corrected suction records throughout the whole test. Accumulated irrigation + rainfall is also shown for reference. 
Iluvia. En el sensor S4 $(15 \mathrm{~cm})$, también se observan caídas de succión posteriores a los eventos de lluvia más intensos, aunque de mucha menor amplitud que la respuesta del sensor superficial. El sensor S10 (35 $\mathrm{cm}$ ) no muestra un comportamiento que pueda correlacionarse con los eventos de lluvia o con los demás sensores. El sensor S5 $(45 \mathrm{~cm})$ muestra una moderada tendencia al secado a lo largo de todo el ensayo, pero aparentemente sin influencia de los fenómenos meteorológicos. Un aspecto notable es que, en general, la succión medida con el sensor $\mathrm{S} 10(35 \mathrm{~cm})$ es siempre mayor a la medida por el sensor $\$ 4(15 \mathrm{~cm})$.

\section{Discusión}

El comportamiento que se observa tanto en los registros de humedad como de succión es complejo. Una dificultad añadida para su interpretación, es que el volumen de medición de los sensores de humedad excede el espesor de muchos de los estratos. Las lecturas obtenidas deben entenderse como un valor medio dentro del volumen de medida que puede abarcar varios tipos de materiales muy diferentes.

\section{Infiltración}

El ensayo de riego en la Fase $A$ y las lluvias de verano en la Fase B del experimento, constituyeron aportes de agua de similar magnitud. El ensayo de riego aportó $125 \mathrm{~mm}$ en tanto que las lluvias de enero de 2013 totalizaron $115 \mathrm{~mm}$. La diferencia importante es que, en el ensayo de riego, esa cantidad de agua se aplicó en sólo 3 días y en el caso de las lluvias en el transcurso de 30 días. En el segundo año de ensayo (2014) la cantidad total de lluvia es algo menor $(93 \mathrm{~mm}$ ) y además esa cantidad resultó distribuida a lo largo de 3 meses (noviembre de 2013 a febrero de 2014). Además de las típicas lluvias torrenciales de verano, se registraron durante el primer año algunas precipitaciones de otoño-invierno, típicamente de menor magnitud e intensidad. Sin embargo, estos eventos menores también se ven reflejados en los tres sensores más superficiales: $\mathrm{S} 1(7 \mathrm{~cm}), \mathrm{S} 6(9 \mathrm{~cm})$ y $\mathrm{S} 9(7 \mathrm{~cm})$, (Fig. 13 y Fig. 17).

Evidentemente, la capa superficial (primeros 10 $\mathrm{cm}$ ) está fuertemente influenciada por los agentes atmosféricos, a pesar de su consistencia dura y baja permeabilidad. Posiblemente tenga una influencia determinante en la infiltración el patrón de pequeñas grietas observado en esta capa. Rodríguez (2002), Rodríguez y Oldecop (2006), observó en ensayos de columna en laboratorio, donde se recrearon las condiciones de vertido de relaves con consistencia de lodo y se indujo la formación de grietas por medio de secado, incrementos de más de un orden de magnitud en la permeabilidad debido a la red de caminos preferenciales creados por grietas e interfaces entre estratos.

Al analizar la respuesta de los sensores instalados a mayor profundidad durante el ensayo de riego (Fig.
11), lo primero que llama la atención es que no reaccionan en un orden consistente con un frente de mojado que avanza hacia abajo. Por el contrario, lo que se observa es que el orden de la respuesta no responde a la posición del sensor, sino más bien al tipo de material del estrato donde está instalado. Así, por ejemplo, los sensores S1 $(7 \mathrm{~cm})$, S6 $(9 \mathrm{~cm})$ y S8 $(75$ $\mathrm{cm}$ ), instalados en estratos de partículas finas (limo y limo arcilloso) reaccionan dos días antes que el resto de los sensores S2 $(15 \mathrm{~cm}), \mathrm{S} 7(20 \mathrm{~cm})$ y S3 $(35 \mathrm{~cm})$, instalados en estratos de arena. Aún dentro de cada uno de estos grupos, se observa por ejemplo que el sensor S6 reacciona antes y de una forma mucho más abrupta que el sensor S1, a pesar que el primero está más profundo. En el grupo de los sensores instalados en arena, el S7 alcanza valores de humedad significativamente mayores que el S2, a pesar que el segundo estaba instalado $5 \mathrm{~cm}$ por arriba del primero. La Figura 14, donde se muestran perfiles de grado de saturación, da cuenta también de este comportamiento.

Estas anomalías constituyen un indicio de flujo heterogéneo durante los procesos de infiltración. Ese flujo heterogéneo pudo estar asociado a grietas, aunque estas sólo se han observado en los $7 \mathrm{~cm}$ superiores del perfil, o a inestabilidad del frente de mojado, fenómeno también conocido en inglés como "fingering". La inestabilidad del frente de mojado puede ocurrir en materiales homogéneos para tasas de riego elevadas. También puede ocurrir en materiales estratificados en donde se alternan materiales de muy diferente tamaño de grano, como ocurre en el caso estudiado. Cada interfaz entre un estrato superior fino (limo o arcilla) y un estrato inferior grueso (arena) constituye una barrera capilar. Para que el frente de mojado pueda atravesar tal barrera, el grado de saturación en el estrato fino debe aumentar de tal manera que la succión caiga a un valor que haga que, en el material grueso, la fase líquida adquiera continuidad. A este valor de succión se lo conoce como valor de entrada de agua (Stormont and Morris, 1998). La acumulación de agua en el estrato fino hace que cualquier mínima heterogeneidad en el material grueso determine la ruptura local de la barrera capilar y se inicie un flujo concentrado a través de la capa gruesa, permaneciendo el resto de la capa con baja saturación y por lo tanto con baja permeabilidad, lo que impide que la zona de flujo se amplíe. Este proceso puede explicar el motivo por el cual el sensor más profundo, $\mathrm{S} 8$, reacciona mucho antes que todos los sensores instalados más arriba, pero en arena.

Un aspecto sorprendente del comportamiento del sensor S8 $(75 \mathrm{~cm})$ es que después de su rápida reacción a los riegos 1 a 8 (Figura 11 y 14), las lecturas tienen un decaimiento sostenido a lo largo de todo el resto del experimento. Si el mecanismo por el cual el agua llegó a la profundidad de este sensor fue el "fingering", entonces implica que a partir del segundo día de ensayo este fenómeno fue suprimido para la posición del sensor S8. Se pueden proponer dos 
explicaciones para ello. Por un lado, es posible que la elevada tasa de riego aplicada en el día 1 indujera el "fingering" y que la intensidad de los riegos y lluvias que siguieron no fue suficiente para volver a provocar el fenómeno, al menos hasta dicha profundidad. Una explicación diferente puede ser que la humedad remanente de los primeros riegos y su uniformización en las capas finas superiores haya hecho más efectivo el efecto de barrera capilar en las múltiples interfaces (entre estratos finos y gruesos) contenidas en el paquete.

Todos los demás sensores, excepto el S8, tienen algún tipo de respuesta a los eventos de lluvia (Fig. 13, Fig. 15 y Fig. 17), menos evidentes y con mayor retardo en los sensores de profundidad intermedia (S2, S3 y S7), que en los superficiales (S1 y S6).

\section{Evaporación}

Durante los periodos del experimento sin riego o lluvia, se observó un decaimiento gradual de las lecturas de los sensores de humedad (Figs. 12, Fig. 13 y Fig. 14). Sin embargo, también en esta situación se pueden distinguir dos grupos de sensores con comportamiento diferente. Los sensores superficiales S1 $(7 \mathrm{~cm}), \mathrm{S} 6(9 \mathrm{~cm}), \mathrm{S} 2(15 \mathrm{~cm})$ y $\mathrm{S} 7(20 \mathrm{~cm})$, tienen el comportamiento típico de un proceso de ascenso capilar y evaporación. Consistentemente, los sensores de succión S9 $(7 \mathrm{~cm})$ y S4 $(15 \mathrm{~cm})$ muestran una evolución compatible con ese tipo de proceso de secado (Fig. 17).

Por otra parte, las lecturas del sensor S5 $(35 \mathrm{~cm})$, se mantuvieron la mayor parte del tiempo fluctuando ligeramente alrededor de un $6 \%$ de humedad, con reacciones a periodos de infiltración o secado muy amortiguadas (Fig. 13). El sensor $S 8(75 \mathrm{~cm})$ no muestra variaciones ni reacciones posteriores a la finalización del ensayo de riego. La onda de largo periodo que se observa en el registro del sensor S8 posiblemente se debe a la variación anual de temperatura.

En principio, resulta difícil pensar que en un ambiente árido donde el déficit hídrico es muy importante (relación evaporación potencial anual / precipitación anual $>10$ ) la atmósfera no pueda extraer la humedad de estratos ubicados a tan solo $35 \mathrm{~cm}$ de profundidad. Sin embargo, los registros de humedad no parecen indicar que las capas a esa o mayor profundidad estén afectadas por la evaporación, al menos en forma permanente, y más bien pareciera que las variaciones de humedad responden a una redistribución de agua o percolación hacia capas más profundas. Los registros de succión parecen confirmar esta hipótesis. El sensor S10 $(35 \mathrm{~cm})$ registra una succión siempre mayor que el $\mathrm{S} 4(15 \mathrm{~cm})$, lo cual imposibilita el ascenso capilar.

La Figura 16 presenta los datos de campo de succión matricial vs. grado de saturación (calculado a partir de las medidas de humedad volumétrica) para pares de sensores instalados a la misma profundidad.
Para referencia se incluyen en la misma figura datos de la curva de retención de la arena limosa (SM) y del limo arcilloso (ML-CL), obtenidos en laboratorio con muestras de Castaño Viejo. También se muestran las curvas que ajustan los datos de laboratorio utilizando la expresión propuesta por van Genuchten (1980). Considerando las incertidumbres que afectan las medidas de succión matricial en el campo y que los sensores de succión y humedad no midieron en el mismo punto, sino que estaban instalados a una distancia horizontal de $50 \mathrm{~cm}$ aproximadamente, la coincidencia entre los datos de campo y de laboratorio es bastante buena. La figura permite visualizar cómo, mientras la capa superficial de limo interactúa con la atmósfera, recorriendo prácticamente todo el rango de su curva de retención, las capas de arena a 15 y $35 \mathrm{~cm}$ de profundidad se mantienen a lo largo de todo el ensayo en la zona de saturación residual. En esa zona de la curva de retención la fase líquida pierde continuidad, lo que causa la interrupción del flujo líquido.

También en este caso, el mecanismo que permite explicar este comportamiento es el de la barrera capilar. En un paquete de estratos alternados de material fino (limo) y grueso (arena), el equilibrio hidráulico determina que la succión de dos estratos debe igualarse en la interfaz. Sin embargo, debido a que sus curvas de retención son radicalmente diferentes, la distribución de humedad cambia a saltos: los limos tienen elevada humedad en tanto las arenas tienen baja humedad. Este efecto ya se observó en el campo (Fig. 7).

Partiendo con una cierta cantidad de agua en el sistema (después de una lluvia, por ejemplo), las capas

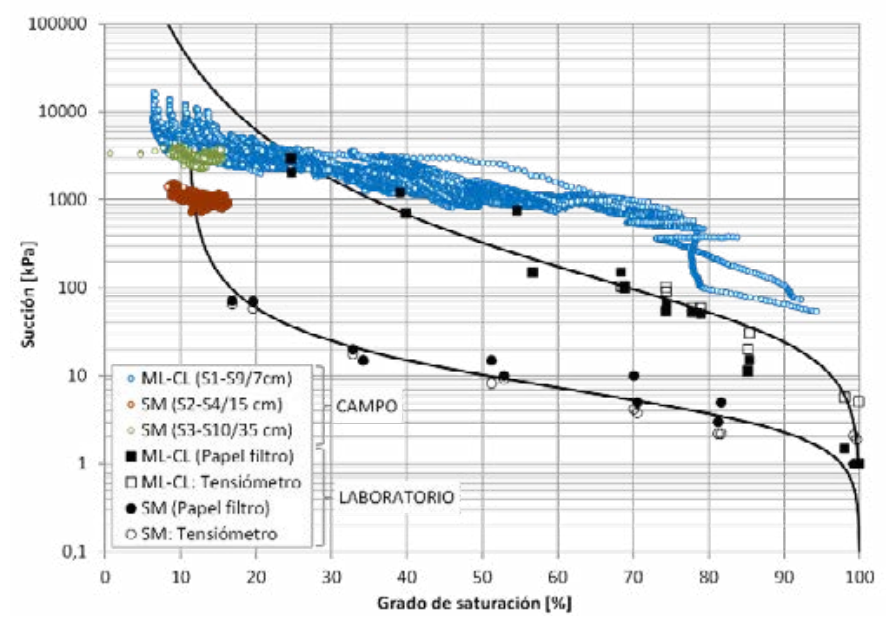

Figura 18. Datos de campo de succión matricial vs grado de saturación, obtenidos con pares de sensores ubicados a igual profundidad, comparados con datos de laboratorio de la curva de retención de materiales similares al de la capa instrumentada. Las líneas continuas corresponden a curvas de van Genuchten (1980) ajustadas a los datos de laboratorio.

Figure 18. Field data of matric suction vs. degree of saturation (from VWC measurements), compared to laboratory retention curve data for similar materials of the instrumented layer. Continuous lines are van Genuchten (1980) curves fitted to the laboratory data. 
de limo absorberían la humedad, secando las capas de arena. Esto causaría que la permeabilidad no saturada de la arena caiga drásticamente, pudiendo llegar a perderse la continuidad la fase líquida. Las capas de arena se convertirían así en barreras para el ascenso capilar y entonces el único transporte posible de agua hacia la atmósfera sería por difusión molecular del vapor. Pero, además, las capas de limo tendrían grados de saturación elevados, por lo que constituirían una barrera para el flujo de gases y también para la difusión de vapor. En este punto el secado se vería totalmente impedido, quedando las capas inferiores completamente aisladas de la atmósfera.

\section{Conclusiones}

Los relaves estudiados presentan un perfil estratigráfico compuesto de capas con espesores variables, comprendidos entre 1 y $100 \mathrm{~mm}$. Se alternan capas de limo (ML), arcilla (CL) y arena muy fina (SM), formando a veces paquetes de estratos muy delgados. La estructura en general es poco densa y esto se debe a la sedimentación de las partículas en presencia de agua. Sin embargo, en la superficie se desarrolla una capa dura o costra formada por limos con intercalaciones de delgados estratos arenosos

Para eventos de lluvia típicos de la zona (incluso para los de intensidad extraordinaria como los de enero de 2013) la infiltración penetra una profundidad limitada, probablemente algo mayor a $35 \mathrm{~cm}$ y menor de $75 \mathrm{~cm}$. Sólo para la intensidad artificialmente elevada del evento de riego, se observó una penetración de la infiltración a $75 \mathrm{~cm}$ o más. Esto último podría ocurrir también en el caso de encharcamiento de agua en la zona de la laguna. El experimento muestra indicios de flujo heterogéneo ("fingering") durante los procesos de infiltración, tanto en los aportes del ensayo de riego como en los producidos por Iluvia. El desarrollo del fenómeno parece aumentar con la intensidad de la lluvia y por tanto también la profundidad de penetración. Por el contrario, cuando el fenómeno de "fingering" no llega a desarrollarse, la profundidad de penetración de la infiltración está limitada por el fenómeno de barrera capilar.

Por otra parte, en las etapas de evaporación, el experimento sugiere que los efectos de secado por este mecanismo no se extienden a más de 20 a $35 \mathrm{~cm}$ de profundidad. La presencia de delgados estratos de arena alternados con estratos de limo conforma un sistema de barrera que resulta extraordinariamente efectivo para evitar la evaporación del agua, tanto por ascenso capilar como por difusión de vapor.

Se puede concluir que, para el caso estudiado, la capa activa donde se desarrollan todos los procesos de intercambio de agua con la atmósfera, tiene una profundidad límite entre $35 \mathrm{~cm}$ y $75 \mathrm{~cm}$. Un dato interesante, que apoya esta conclusión, se desprende de la observación del perfil estratigráfico (Fig. 9 y Fig. 10). La oxidación intensa de algunos estratos finos se observa sólo hasta los $50 \mathrm{~cm}$ de profundidad, lo cual constituye un indicador independiente de la profundidad de la capa activa. El agua de lluvia transporta el oxígeno disuelto necesario para la oxidación. Por debajo de los $50 \mathrm{~cm}$ se observa sólo oxidación muy ligera, lo que sugiere que sólo ocasionalmente el agua de lluvia penetra por infiltración a más de $50 \mathrm{~cm}$ en el perfil vertical.

La particularidad del sistema estudiado es que su funcionamiento parece ser asimétrico. La penetración del agua durante los procesos de infiltración es más profunda que la extensión de los efectos de secado por evaporación. A largo plazo, esto produciría un ingreso neto de agua al depósito, que alcanzando las capas profundas ya no podría ser extraída por la atmósfera. Esto lo convierte en un sistema que tiende a atrapar agua, produciendo un efecto tipo invernadero sobre el balance de agua. Esta conclusión es consistente con la gran cantidad de agua que se ha encontrado almacenada en este tipo de depósitos aun habiendo transcurrido periodos de tiempo muy prolongados desde su abandono (Oldecop et al., 2011).

El vertido de relaves en consistencia de lodo genera estructuras abiertas y poco densas que por efecto de la consolidación debería alcanzar cierto grado de compacidad y resistencia. Sin embargo ensayos de campo realizados en PR abandonadas arrojan resistencias extremadamente bajas, con números de golpes del ensayo de penetración estándar inferior a 4 (Garino et al., 2017; IGME, 2004).

Si se tiene en cuenta además que el grado de saturación permanece elevado en el interior de los relaves, resulta probable que se presenten problemas de estabilidad si estas estructuras se ven sometidas a acciones dinámicas debidas a sismos de moderada intensidad (licuación).

Por lo expuesto, resulta conveniente a la hora de realizar análisis de estabilidad estructural a largo plazo considerar a los relaves, salvo una capa superficial, con resistencias bajas y en condición cercana a la saturación, aun cuando las condiciones ambientales sean propicias para la evaporación.

Por otro lado resulta apropiado considerar parámetros de resistencia post-licuación o residuales a los efectos de verificar estabilidad en zonas sísmicamente activas.

\section{Referencias}

Alonso E.E. and Gens A. 2006. Aznalcóllar dam failure. Part 1: Field observations and material properties. Géotechnique, 56(3):165-183.

Bligth G. E. 1997. Destructive mudflows as a consequence of tailing dyke failures. Proceedings of the Institution of Civil Engineers, 125: 9-18.

Bligth G. E. and Fourie A. B. 2005. Catastrophe revisited - disastrous flow failures of mine and municipal solid waste. Geotechnical and Geological Engineering, 23:219-248. 
Bray J. D. and Frost, J. D. 2010. Geo-Engineering Reconnaissance of the 2010 Maule, Chile Earthquake, a report of the NSF-sponsored GEER Association Team, http://www.geerassociation.org/.

Bowker L.N. and Chambers D.M. 2015. The Risk, Public Liability, and Economics ofTailings Storage Facility Failures. Earthwork Act, 1-56.

Chandler R. J. andTosatti G. 1995. The Stava dams failure, Italy, July, 1985. Proceedings of the Institution of Civil Engineers, 113:67-79.

Davies M. P. 2002. Tailings Impoundment Failures: Are Geotechnical Engineers Listening?. Geotechnical News, 20:31-36.

Dorigo, W.A., Xaver, A., Vreugdenhil, M., Gruber, A., Hegyiová, A., Sanchis-Dufau, A. D., Zamojski, D., Cordes, C., Wagner, W., Drusch, M. 2013. Global automated quality control of in situ soil moisture data from the International Soil Moisture Network. Vadose Zone Journal, 12(3). http://dx.doi.org/10.2136/ vzj2012.0097.

Fourie A.B., Blight G.E., Papageorgiou G. 2001. Static liquefaction as a possible explanation for the Merriespruit tailings dam failure. Canadian Geotechnical Journal, 38:707-719.

García C. 2004. Impacto y riesgo ambiental de los residuos minero-metalúrgicos de la Sierra de Cartagena-La Unión (Murcia-España). Tesis Doctoral, Universidad Politécnica de Cartagena.

Garino L., Rodari G., Oldecop L. 2017. Characterization of mine waste materials after 50 years of climate interaction. Second Pan-American Conference on Unsaturated Soils, Dallas, United States.

Gramage R. 1983. Estudio geológico minero de las vetas Compañía y Flor de Castaño, Distrito Minero Castaño Viejo, Pcia. de San Juan. Trabajo Final de la Carrera Licenciatura en Ciencias Geológicas. Facultad de Ciencias Exactas, Físicas y Naturales. Universidad Nacional de San Juan, Argentina.

Harder L.F. and Stewart, J.P. 1996. Failure of Tapo Canyon Tailings Dam. Journal of Performance of Constructed Facilities, ASCE, 10 (3): 109-114.

ICOLD 2001. Tailings dams risk of dangerous occurrences, lessons learnt from practical experiences, Bulletin 121, United Nations Environmental Programme (UNEP) Division of Technology, Industry and Economics (DTIE) and International Commission on Large Dams (ICOLD), Paris, 2001.

IEEIRP 2015. Report on Mount Polley Tailings Storage Facility Breach. Independent Expert Engineering Investigation and Review Panel. January 30, 2015.

IGME 2004. Evaluación de riesgos y definición de medidas correctoras en depósitos de lodos abandonados procedentes de procesos de tratamiento de actividades extractivas en la región de Murcia. Aplicación a los depósitos "San Cristóbal" (N ${ }^{\circ}$ 0976-3-0006 y 0976-3-0005). Instituto Geológico y Minero de España, 2004.

Kapilaratne, R. J. and Lu, M. 2017. Automated general temperature correction method for dielectric soil moisture sensors. Journal of Hydrology, 551:2032016. https://doi.org/10.1016/j.jhydrol. 2017.05.050

Malmberg, C. G. and Maryott, A. A. 1956. Dielectric constant of water from $0^{\circ}$ to $100^{\circ} \mathrm{C}$. Journal of Research of the National Bureau of Standards, 56:1-8.

Morgenstern N.R., Vick S.G., Viotti C.B., Watts B.D. 2016. Fundão Tailings Dam Review Panel. Report on the Immediate Causes of the Failure of the Fundão Dam.

Oldecop L. y Rodríguez R. (2006). Estabilidad y seguridad de depósitos de residuos mineros. Los residuos minero-metalúrgicos en el medio ambiente. Editorial Instituto Geológico y Minero de España, 197-244.

Oldecop L., Zabala F., Rodríguez R., Garino L. 2008. Funcionamiento hidráulico, estabilidad y mecanismos de rotura de presas de relaves mineros. $\mathrm{V}$ Congreso Argentino de Presas y Aprovechamientos Hidroeléctricos. Tucumán, Argentina.

Oldecop L., Garino L., Muñoz, J.J., Rodríguez, R., García C. 2011. Unsaturated behavior of mine tailings in low precipitation areas. Unsaturated soils, 14251430. CRC Press/Balkema, ISBN: 9780415604284. http://dx.doi.org/10.13140/RG.2.1.4660.7527.

Or, D. and Wraith, J. M. 1999. Temperature effects on soil bulk dielectric permittivity measured by time domain reflectometry: A physical model. Water Resources Research, 35:371-383.

Ramírez M., Salinas L. Carrascosa H., Negrelli M. 2002. Caracterización y evaluación de las presas mineras del Distrito Castaño Viejo, Calingasta, San Juan. II Congreso Argentino de Presas y Aprovechamientos Hidroeléctricos, San Juan, Argentina.

Rico M., Benito G., Salgueiro A.R., Díez-Herrero A., Pereira H.G. 2008. Reported tailings dam failures - A review of the European incidents in the worldwide context. Journal of Hazardous Materials, 152:846852.

Roche C., Thygesen K., Baker E. 2017. Mine Tailings Storage: Safety Is No Accident. A UNEP Rapid Response Assessment. United Nations Environment Programme and GRID-Arendal, Nairobi and Arendal, www.grida.no.

Rodríguez R. 2002. Estudio experimental de flujo y transporte de cromo, níquel y magnesio en residuos de la zona minera de Moa (Cuba): influencia del comportamiento hidromecánico. PhD Thesis, Universitat Politècnica de Catalunya, Barcelona.

Rodríguez R. y Oldecop L. 2006. Propiedades físicas, mecánicas e hidrogeológicas de los residuos minero-metalúrgicos sólidos. Los residuos minero-metalúrgicos en el medio ambiente. Editorial Instituto Geológico y Minero de España., Madrid, 2006. ISBN 84-7840-656-5

Seyfried, M. S. and Grant, L. E. 2007. Temperature effects on soil dielectric properties measured at 50 MHz. Vadose Zone Journal, 6:759-765.

Stormont J.C. and Morris C. E. 1998. Method to estimate water storage capacity of capillary barriers. 
Journal of Geotechnical and Geoenvironmental Engineering, 124 (4): 297-302.

van Genuchten M.Th. 1980. A closed-form equation for predicting the hydraulic conductivity of unsaturated soils. Soil Science Society of America Journal, 44:892-898.

Walthert, L. and Schleppi P. 2018. Equations to compensate for the temperature effect on readings from dielectric Decagon MPS-2 and MPS-6 water potential sensors in soils. Journal of Plant Nutrition and Soil Science, 181:749-759. https://doi. org/10.1002/jpln.201700620

Zandarín M. T., Oldecop L., Rodríguez R., Zabala F. 2009. The role of capillary water in the stability of tailing dams. Engineering Geology, 105 (1-2):108118.

Recibido: julio 2020

Revisado: diciembre 2020

Aceptado: enero 2021

Publicado: diciembre 2021 
